INTEGRATED PAPER

\title{
Fundamental Aspects of Gas Phase Ion Chemistry Studied Using the Selected Ion Flow Tube Technique
}

\author{
Shuji Kato*
}

(Received May 6, 2005; Accepted May 17, 2005)

\begin{abstract}
Gas phase ion chemistry using the selected ion flow tube (SIFT) technique is discussed with the focus on fundamental studies on ion structure, energetics, reactivity, and dynamics: Laser-induced fluorescence (LIF) kinetics/dynamics of $\mathrm{N}_{2}{ }^{+}(v)$ ions (vibrationally state-selected ion-molecule reactions; energy transfer processes), mechanisms of prototypical organic ion reactions $\left(\mathrm{S}_{\mathrm{N}} 2\right.$ nucleophilic substitution and kinetic isotope effects; hydrogen/deuterium exchange; isotope effects in termolecular association), and ion-molecule reactions with biological implications $\left(\mathrm{S}_{\mathrm{N}} 2\right.$ reactions at a nitrogen center; $\mathrm{E}_{\mathrm{CO}} 2$ elimination reactions of alkyl hydroperoxides). Anion chemistry of small nitroalkanes is also presented. A powerful combination of SIFT and negative ion photoelectron spectroscopy is used to study thermochemistry and structure of energetic and exotic ions (alkyl peroxides; anionic derivatives of diazo and azole compounds, cyclooctatetraene, and cyclopentanone). Finally, some of the new directions in SIFT research are discussed, i.e., reactions of ions with polyatomic organic radicals, and chemical ionization mass spectrometry with new detection schemes.
\end{abstract}

\section{Introduction}

The selected ion flow tube (SIFT) is an MS-MS technique to investigate chemical reactions of massselected ions in a flowing afterglow environment.1) Mass-selected ions are thermalized by collision with buffer gas molecules (e.g., helium) prior to reaction with a neutral reagent added. Compared to conventional ion beam techniques, low collision energies with the SIFT technique allow studies of ion-molecule reactions under truly thermal conditions $\left(300 \mathrm{~K}\right.$ or $E_{\mathrm{cm}} \approx$ $0.04 \mathrm{eV})$. The technique was originally developed by Smith and Adams at Birmingham. ${ }^{2), 3)}$ A later review ${ }^{4)}$ described some additional features to the original design, e.g., the VT-SIFDT (variable temperatureselected ion flow drift tube) apparatus to explore different collision-energy regimes. Graul and Squires ${ }^{5)}$ thoroughly and comprehensively reviewed flow reactor techniques in general, including SIFT. The technique later incorporated laser-induced fluorescence ${ }^{6)}$ and triple quadrupole ${ }^{7)}$ detections for extended capabilities.

This article discusses the SIFT ion chemistry work at Colorado, with the focus on fundamental studies which the author has conducted in recent years. Following a brief description of the instrument, studies of vibrationally state-selected ion-molecule reactions and energy transfer processes are presented as examples of the most fundamental aspects of gas phase ion chemistry. Nucleophilic substitution $\left(\mathrm{S}_{\mathrm{N}} 2\right)$ reactions and associated kinetic isotope effects, hydrogen/deuterium exchange reactions, and isotope effects in termolecular association reactions are subsequently discussed, with an emphasis on how isotope labeling can be used to probe the structure and dynamics of ion-neutral inter-

* Department of Chemistry and Biochemistry, University of Colorado (Boulder, CO 80309-0215, USA)

E-mail: Shuji.Kato@colorado.edu mediate complexes. These sections are followed by discussions of individual ion chemistry in two categories. First, there are ion-molecule reactions with strong biological implications: $\mathrm{S}_{\mathrm{N}} 2$ reactions at a nitrogen center and a novel $\mathrm{E}_{\mathrm{CO}} 2$ type of elimination reactions. Secondly, the technique has been used extensively to determine the thermochemistry and structure of energetic and exotic ions. The article concludes by presenting some of the most recent projects and new directions that are being pursued with the SIFT technique, i.e., reactions of ions with polyatomic organic radicals and new schemes for chemical ionization mass spectrometry.

\section{SIFT Instrument}

The selected ion flow tube apparatus used at Colorado is schematically illustrated in Fig. 1. Details of the design and operation have been given elsewhere. ${ }^{6), 8)}$ Ions are produced by using simple electron impact on neutral precursors or sequential ion-molecule reactions in the source flow tube containing helium $(\sim 0.3$ Torr $)$. They are selected by the SIFT quadrupole mass filter and injected through an injection orifice into the second flow tube containing the helium buffer gas $(0.5$ Torr, $\approx 300 \mathrm{~K})$. The SIFT injection energy $\left(E_{\text {inj }}\right)$, which is defined by the potential difference between the source flow tube and the injection orifice, is typically set to $20 \mathrm{eV}$ in order to cleanly inject the reagent ions with negligible fragmentation. Injected ions are quickly thermally equilibrated to $300 \mathrm{~K}$ by collisions with the helium buffer gas. A laminar flow of He is established after the initial turbulent flow regime $(30-40 \mathrm{~cm}$ in length). The ions entrained in He are then allowed to react with neutral gases added through multiple reactant inlets placed along the reaction flow tube. The length of the laminar region $(\approx 1 \mathrm{~m})$ and the flow velocity $\left(\approx 100 \mathrm{~ms}^{-1}\right)$ gives a maximum reaction time of about $10 \mathrm{~ms}$. Parent and product ions are analyzed 


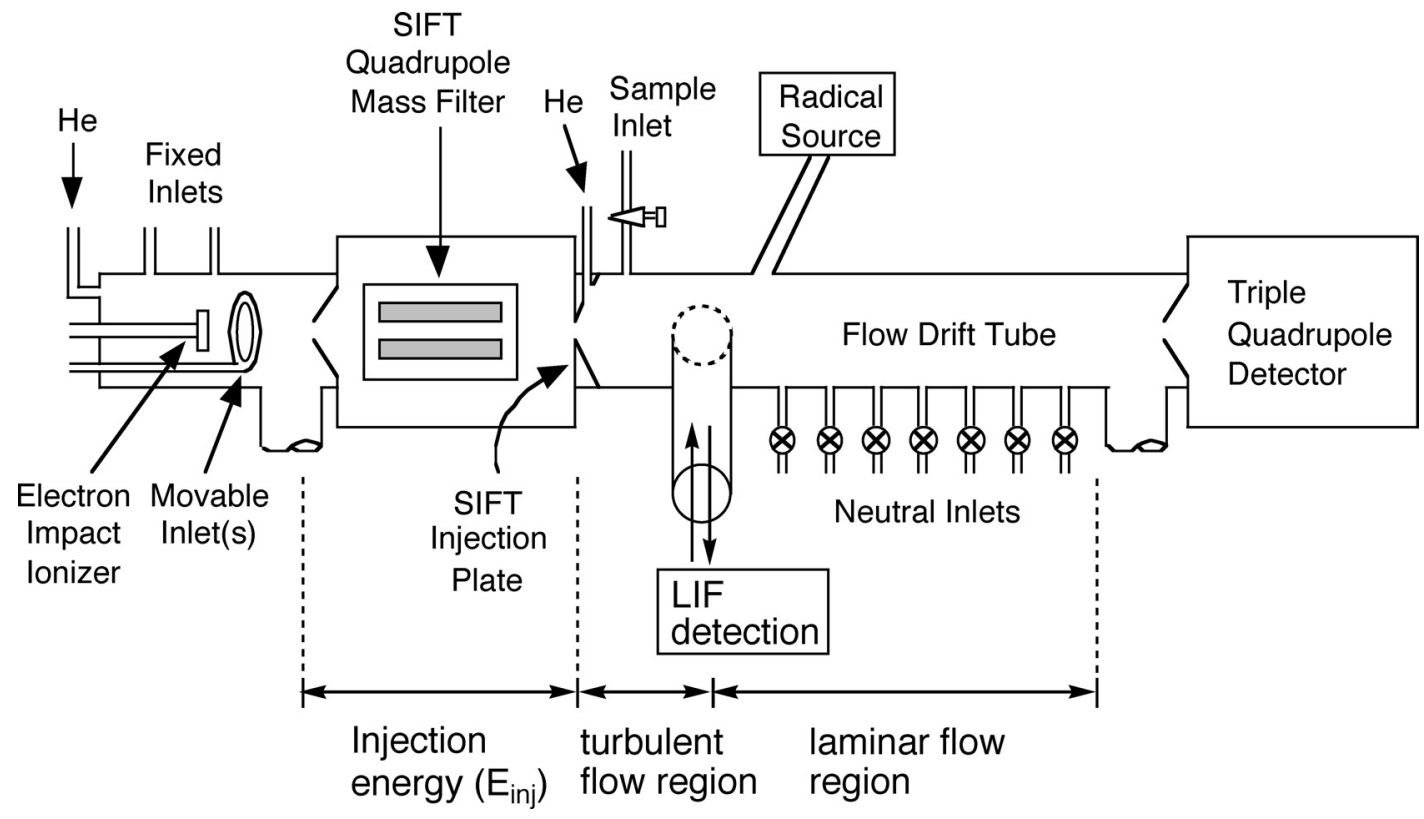

Fig. 1. The tandem flowing afterglow SIFT instrument.

with a detection quadrupole mass filter placed at the end of the flow tube. Absolute reaction rate constants are measured by monitoring the kinetic decay of the parent ions while varying the reaction distance, i.e., the neutral inlet positions. The detector has a triple quadrupole capability that allows studies of collisioninduced dissociation (CID) of ions. Alternatively, unimolecular dissociation can be effected by SIFT injection of ions at higher $E_{\text {inj }}$; in this case ion dissociation takes place near the injection orifice in the high-pressure $\mathrm{He}$ environment. This mode of experiment ("SIFT-CID") involves multiple ion-He collisions, thus producing qualitative and yet informative results with high sensitivity. An additional feature with the SIFT instrument is the drift section in the second flow tube (Fig. 1). Ion mobilities and reactions at higher kinetic energies (up to $E_{\mathrm{cm}} \sim 1 \mathrm{eV}$ ) are examined in the electric field applied along the flow tube. ${ }^{9), 10)}$

Compared to polyatomic ions, vibrational relaxation of small diatomic ions is extremely slow in He. Thus, ion chemistry of vibrationally excited ions can also be explored. The capability of the SIFT technique has been extended by incorporating high-resolution spectroscopic detection with laser induced fluorescence (LIF, Fig. 1). ${ }^{6), 11)}$ The SIFT-LIF technique allows studies of vibrationally state selected ion chemistry, e.g., vibrational specificity and energy transfer in ion-molecule reactions, at thermal energy $\left(E_{\mathrm{cm}} \approx 0.04 \mathrm{eV}\right)$. The optical detection has a significant advantage over the conventional monitor ion technique, ${ }^{12)}$ which chemically probes for vibrationally excited states using reactions that are fortuitously specific to certain excited states, e.g., $\mathrm{N}_{2}{ }^{+}(v>0)+\mathrm{Ar} \rightarrow \mathrm{N}_{2}+\mathrm{Ar}^{+}$without isotope specificity. Reactant ions are generated by electron impact in various vibrational states, and immediately extracted into the SIFT quadrupole chamber before collisional relaxation takes place. The ions are then mass selected and injected into the second flow tube, where they are rapidly thermalized translationally and rotationally while retaining vibrational excitation. Following a reaction with a reagent added through the sample inlet (Fig. 1), the reactant and product ion vibrational states are monitored using LIF. There are requirements for SIFT-LIF experiments to be feasible:6), 11) Vibrationally excited reagent ions need to be readily generated and stably injected at high densities. Collisional quenching of vibrationally excited states must be sufficiently slow with the He buffer gas (although slow quenching with He can also be measured; see below) and the radiative quenching be also negligible within the reaction time scale of about $150 \mu$ s before the LIF detection. ${ }^{6}$ Small, non-polar $\mathrm{N}_{2}{ }^{+}(v)$ ions have thus been studied extensively with this technique using the $\mathrm{B}-\mathrm{X}$ electronic transition for LIF.

\section{Vibrationally State-Selected Ion Chemistry}

\subsection{Vibrational energy transfer and relaxation}

Vibrational relaxation of ions in collision with rare gas atoms (He, Ne, Ar, Kr, Xe) is slow. The ion-neutral interaction is relatively weak because the rare gas atoms have no permanent dipoles or chemical interactions with ions. Relaxation via vibration-to-vibration $(\mathrm{V}-\mathrm{V})$ or vibration-to-rotation (V-R) energy transfer does not occur with monatomic rare gas molecules, and only the vibration-to-translation $(\mathrm{V}-\mathrm{T})$ process is available for the disposal of ion vibrational energy. Compared to intermolecular potential wells for neutral-neutral systems, however, charge-induced dipole interactions facilitate deep potential wells for ion-neutral systems (typically $\sim 0.2 \mathrm{eV}$ ). As the consequence, ion vibrational relaxation is generally more efficient than the corresponding neutral relaxation. ${ }^{12)} \mathrm{V}$-T energy transfer/relaxation with rare gas atoms is the simplest case to examine the effects of intermolecular potentials during ion-neutral versus neutral-neutral collisions. Because of the inherent experimental difficulties, ion vibrational relaxation has been considerably less explored than is relaxation of neutral molecules. ${ }^{13)-15)}$

We used the SIFT-LIF technique to measure vibrational relaxation of $\mathrm{N}_{2}{ }^{+}(v)$ ions with rare gas atoms. ${ }^{16}$ ) 


$$
\begin{gathered}
\mathrm{N}_{2}{ }^{+}(v)+\mathrm{M} \stackrel{k_{q}(v)}{\longrightarrow} \mathrm{N}_{2}{ }^{+}\left(v^{\prime}<v\right)+\mathrm{M} \\
\stackrel{k_{\mathrm{CT}}(v)}{\longrightarrow} \mathrm{N}_{2}+\mathrm{M}^{+}
\end{gathered}
$$

The optical detection allows individual vibrational levels of $\mathrm{N}_{2}{ }^{+}(v)$ to be monitored. At an injection energy of $E_{\text {inj }}=49 \mathrm{eV}$, initial vibrational population distributions of $\mathrm{N}_{2}{ }^{+}(v)$ were determined by LIF to be $46 \%(v=0)$, $23 \%(v=1), 15 \%(v=2), 12 \%(v=3), 4 \%(v=4)$, and $<1 \%$ $(v=5) .{ }^{17)}$ Following addition of reagent rare gases, evolution of these states can be measured and analyzed, and vibrationally specific quenching rate constants $k_{\mathrm{q}}(v)$ determined (Eq. (1)). In practice, measuring slow rate constants is challenging because the measurement can easily be biased by experimental artifacts arising from trace impurities and ion mobility changes upon addition of large amounts of reagents. Also, there are concurrent charge transfer reactions (Eq. (2)) with Ar (for $v>0$ ), $\mathrm{Kr}$, and $\mathrm{Xe}$. Conventional mass spectrometry, on the other hand, is blind to the quenching process in Eq. (1) but can detect the charge transfer products in Eq. (2) as well as impurity reactions. We thus employed a powerful combination of SIFT-LIF and mass spectrometry to unambiguously determine the ion-rare gas relaxation rate constants. ${ }^{16)}$

The measured rate constants $k_{q, 10}$ for vibrational relaxation of $\mathrm{N}_{2}{ }^{+}(v=1) \rightarrow \mathrm{N}_{2}{ }^{+}(v=0)$ are very small; $9.8( \pm$ $0.7) \times 10^{-16}, 3.6( \pm 0.5) \times 10^{-14}$, and $1.6( \pm 0.2) \times 10^{-13}$ $\mathrm{cm}^{3} \mathrm{~s}^{-1}$ for $\mathrm{He}, \mathrm{Ne}$, and Xe, respectively. ${ }^{16)}$ Upper limits were obtained for $\mathrm{Ar}\left(<1.2 \times 10^{-11} \mathrm{~cm}^{3} \mathrm{~s}^{-1}\right)^{18)}$ and $\mathrm{Kr}$ $\left.\left(<2 \times 10^{-12} \mathrm{~cm}^{3} \mathrm{~s}^{-1}\right) .{ }^{16}\right)$ These values are one to two orders of magnitude smaller than previous measurements, which presumably were affected by impurities (reviewed in Kato et al.). ${ }^{16)}$ Specifically, vibrational relaxation with Ar was measured for the first time using LIF. This process cannot be studied with the conventional monitor technique because Ar itself is the only monitor species available to distinguish between $\mathrm{N}_{2}{ }^{+}(v=0)$ and $\mathrm{N}^{+}(v>0)$. It is confirmed that $\mathrm{N}^{+}(v>0)+$ Ar charge transfer is an excellent monitor reaction; vibrational relaxation is negligibly slow compared to the fast charge transfer $\left(k_{\mathrm{CT}}(v>0)=4 \times 10^{-10} \mathrm{~cm}^{3} \mathrm{~s}^{-1}\right)$.

For comparison with neutral-neutral vibration relaxation, the rate constant $k_{q, 10}$ is converted to the deactivation probability per collision, $\langle P\rangle\left(\equiv k_{q, 10} / k_{\mathrm{col}}\right.$, where $k_{\text {col }}$ is the collision rate constant). The values of $\langle P\rangle$ are extremely small, ranging from $1.7 \times 10^{-6}(\mathrm{He})$ to $8.4 \times 10^{-5}(\mathrm{Ne})$ and $1.6 \times 10^{-4}(\mathrm{Xe})$ for collisions with $\mathrm{N}_{2}{ }^{+}(v=1){ }^{16)}$ The inefficient relaxation is reasonable because the $\mathrm{N}_{2}{ }^{+}$ion $\left(\omega_{\mathrm{e}}=2207 \mathrm{~cm}^{-1}\right)$ has to dissipate the large vibrational energy solely into translation. The small probabilities imply that $\mathrm{N}_{2}{ }^{+}(v)$ relaxation proceeds via transfer of a single vibrational quantum per collision, i.e., $v^{\prime}=v-1$, in accord with LandauTeller theory for V-T energy transfer established for neutral-neutral collisions at thermal energy. ${ }^{13), 14)}$ There is a marked difference observed between relaxations of $\mathrm{N}_{2}{ }^{+}(v)$ and $\mathrm{N}_{2}(v)$ however. Measured or estimated values of $\langle P\rangle$ for neutral $\mathrm{N}_{2}(v)$ are $1.6 \times 10^{-8}$ $(\mathrm{He}), 6 \times 10^{-13}(\mathrm{Ne})$, and $1 \times 10^{-14}(\mathrm{Ar})$; $^{16)}$ relaxation is more efficient for lighter collision partners. This trend is consistent with Landau-Teller theory for V-T energy transfer, which assumes the relaxation to occur at the repulsive part of the intermolecular potential. In contrast, observed $\mathrm{N}_{2}^{+}(v)$ ion relaxation is more efficient for heavier collision partners and has a positive correlation with the rare gas polarizability. The magnitudes of the deactivation probability are also greater for $\mathrm{N}_{2}{ }^{+}$ $(v)$ than for $\mathrm{N}_{2}(v)$. The attractive, charge-induced dipole interaction must play a significant role in the $\mathrm{N}_{2}{ }^{+}(v)$ ion vibrational relaxation. We discussed the results in terms of direct versus complex-formation mechanisms. ${ }^{16)}$

The SIFT-LIF technique was extended to studies of $\mathrm{N}_{2}{ }^{+}(v)$ vibrational relaxation/energy transfer with diatomic molecules $\mathrm{N}_{2}{ }^{19)-21)} \mathrm{O}_{2},{ }^{18), 21)} \mathrm{CO}$ and $\mathrm{NO},{ }^{22)}$ and $\mathrm{H}_{2} \cdot{ }^{23)}$ In addition to $\mathrm{V}$-T energy transfer, relaxation via $\mathrm{V}-\mathrm{V}$ and $\mathrm{V}-\mathrm{R}$ processes is possible for these diatomics; the vibrational constants $\omega_{\mathrm{e}}$ are $2359 \mathrm{~cm}^{-1}\left(\mathrm{~N}_{2}\right), 1580$ $\mathrm{cm}^{-1}\left(\mathrm{O}_{2}\right), 2170 \mathrm{~cm}^{-1}(\mathrm{CO}), 1904 \mathrm{~cm}^{-1}(\mathrm{NO})$, and 4395 $\mathrm{cm}^{-1}\left(\mathrm{H}_{2}\right)$ in comparison to $2207 \mathrm{~cm}^{-1}\left(\mathrm{~N}_{2}^{+}\right)$. The attractive potential wells are especially deeper $(\sim 1 \mathrm{eV})$ with $\mathrm{N}_{2}, \mathrm{O}_{2}$, and $\mathrm{NO}$ due to the electron-exchange interactions. ${ }^{24), 25)}$ Efficient relaxation of $\mathrm{N}_{2}{ }^{+}(v)$ is thus expected for these reactants.

Rate constants $k_{q}$ and probabilities $\langle P\rangle$ were determined for $\mathrm{N}_{2}{ }^{+}(v)$ vibrational relaxation, in competition with concurrent charge transfer $\left(\mathrm{N}_{2}, \mathrm{O}_{2}, \mathrm{CO}, \mathrm{NO}\right)$ and hydrogen atom transfer $\left(\mathrm{N}_{2}{ }^{+}+\mathrm{H}_{2} \rightarrow \mathrm{N}_{2} \mathrm{H}^{+}+\mathrm{H}\right)$. As expected, vibrational relaxation is generally more efficient with the diatomic molecules by orders of magnitude than with rare gas atoms, but there are interesting variations found among the different diatomics. Vibrational relaxation with $\mathrm{H}_{2}$ is noticeably slow and only an upper limit of 0.06 was obtained for $\langle P\rangle$, on average for all vibrational levels of $\mathrm{N}_{2}{ }^{+}(v>0) .{ }^{23)}$ This may be reasonable in light of the poor vibrational energy match between $\mathrm{N}_{2}{ }^{+}$and $\mathrm{H}_{2}$ and also the small polarizability of $\mathrm{H}_{2}$.

An intriguing comparison is made between $\mathrm{N}_{2}{ }^{+}(v)+$ $\mathrm{NO}$ and $\mathrm{N}_{2}{ }^{+}(v)+\mathrm{CO}^{22)}$ On average for $v>0$, vibrational quenching is efficient for $\mathrm{NO}$ with $\langle P\rangle$ of 0.22 whereas the deactivation probability is only 0.04 for $\mathrm{CO}$, even though $\mathrm{CO}$ and $\mathrm{N}_{2}{ }^{+}$has a nearly perfect vibrational energy match for resonant $\mathrm{V}-\mathrm{V}$ energy transfer. This suggests that ion vibrational relaxation does not always follow the resonance principles that are well known for neutrals. ${ }^{15)}$ Instead, the lifetime of energized adducts is a more important factor than is the V-V energy gap. The $\mathrm{N}_{2}{ }^{+}(v)+\mathrm{NO}$ system is likely to have a deep attractive well due to the near energyresonant, electron-exchange interaction between $\mathrm{N}_{2}{ }^{+}$ and electronically excited $\mathrm{NO}^{+}\left(a^{3} \Sigma^{+}\right) .{ }^{25)}$ Efficient vibrational energy transfer may occur during the long complex lifetime. Relaxation of $\mathrm{N}_{2}^{+}(v)$ with $\mathrm{O}_{2}$ is also efficient and vibrational-level dependent, the value of $\langle P\rangle$ increasing from $0.15(v=1)$ to $0.30(v=4){ }^{21)} \mathrm{A}$ similar electron-exchange interaction with the excited $\mathrm{O}_{2}{ }^{+}\left(a^{4} \Pi_{\mathrm{u}}\right)$ state has been suggested. ${ }^{25}$ Tachibana et al. ${ }^{26)}$ proposed vibrational relaxation within the stable quartet $\left[\mathrm{N}_{2} \cdot \mathrm{O}_{2}\right]^{+}$complex, which is computationally shown to be formed from $\mathrm{N}_{2}{ }^{+}$and $\mathrm{O}_{2}$ and correlate with $\mathrm{N}_{2}+\mathrm{O}_{2}{ }^{+}\left(a^{4} \Pi_{\mathrm{u}}\right)$.

The SIFT-LIF technique revealed another novel aspect in ion-molecule interactions: multiquantum vibrational transfer/deactivation in collision of $\mathrm{N}_{2}{ }^{+}(v)$ 
with $\mathrm{N}_{2}$ and $\mathrm{O}_{2} \cdot{ }^{11), 19)-21)}$ The $\left[\mathrm{N}_{2} \cdot \mathrm{N}_{2}\right]^{+}$adduct formed from $\mathrm{N}_{2}{ }^{+}+\mathrm{N}_{2}$ is strongly bound by about $1.1 \mathrm{eV} .{ }^{27), 28)}$ In collision of vibrationally excited ${ }^{15} \mathrm{~N}_{2}{ }^{+}(v=2)$ ions with ${ }^{14} \mathrm{~N}_{2}$, the reactant and product ion vibrational states were distinguished by high-resolution LIF with isotope specificity. We observed simultaneous transfer of charge and vibration in this near-energy resonant charge transfer reaction (Eq. (3)). ${ }^{19)}$ Most notably, two vibrational quanta are transferred efficiently in a single collision.

$$
\begin{aligned}
{ }^{15} \mathrm{~N}_{2}{ }^{+}(v=2)+{ }^{14} \mathrm{~N}_{2}\left(v^{\prime}=0\right) \rightarrow \\
{ }^{15} \mathrm{~N}_{2}(v=0)+{ }^{14} \mathrm{~N}_{2}{ }^{+}\left(v^{\prime}=2\right)
\end{aligned}
$$

A detailed kinetic analysis including all reactant vibrational states $(v=0-4)$ indicates that approximately $10 \%$ of the initial ${ }^{15} \mathrm{~N}_{2}{ }^{+}(v=2)$ forms ${ }^{14} \mathrm{~N}_{2}{ }^{+}\left(v^{\prime}=2\right)$ (Eq. (3)) and, by symmetry, another $10 \%$ forms ${ }^{14} \mathrm{~N}_{2}\left(v^{\prime}=2\right)$ neutral (plus $\left.{ }^{15} \mathrm{~N}_{2}{ }^{+}(v=0)\right) .{ }^{21)}$ The observation that the $\mathrm{N}_{2}{ }^{+}$ $(v=2)$ ion relaxes via $|\Delta v|=2$ transitions with such a high efficiency $(\langle P\rangle \approx 0.2)$ is a marked result. For neutral-neutral collisions, only the $|\Delta v|=1$ transition is allowed at thermal energy to first order within the framework of Landau-Teller theory. ${ }^{13), 14)}$

A possible mechanism is direct $\mathrm{V}-\mathrm{V}$ energy transfer between the high-frequency moieties in the long-lived $\left[{ }^{15} \mathrm{~N}_{2}-{ }^{14} \mathrm{~N}_{2}\right]^{+}$complex, as inferred from the similar vibrational constants for $\mathrm{N}_{2}{ }^{+}$and $\mathrm{N}_{2}$. Alternatively, multiquantum vibrational transfer may result from nearresonant, intracomplex charge-exchange transitions $\left[{ }^{15} \mathrm{~N}_{2}{ }^{+} \ldots{ }^{14} \mathrm{~N}_{2}\right] \longleftrightarrow\left[{ }^{15} \mathrm{~N}_{2} \cdot{ }^{14} \mathrm{~N}_{2}{ }^{+}\right]$. Extensive quantum mechanical calculations of the state-to-state cross sections $^{29)}$ support the importance of $|\Delta v|=2$ transitions in thermal energy $\mathrm{N}_{2}{ }^{+}(v)+\mathrm{N}_{2}$ collisions, along with facile single-quantum V-V and V-T processes which were also confirmed from analysis of the LIF kinetics. ${ }^{21)}$

Multiquantum vibrational transfer/relaxation was also indicated in the $\mathrm{N}_{2}{ }^{+}(v)+\mathrm{O}_{2}$ reaction from SIFTLIF rate kinetics for $\mathrm{N}_{2}{ }^{+}(v=0-4) .{ }^{21)}$ As noted above, a deep potential well facilitated by the intermediacy of electronically excited $\mathrm{O}_{2}{ }^{+}\left(a^{4} \Pi_{\mathrm{u}}\right)$ must be responsible for the observation. By comparison, ion deactivation proceeds exclusively via the $|\Delta v|=1$ transition for $\mathrm{NO}^{+}$ $(v)+\mathrm{M}$ systems, ${ }^{30)}$ which have shallow potential wells. In this vein, deactivation of $\mathrm{N}_{2}{ }^{+}(v)+\mathrm{NO}$ may also proceed via multiquantum vibrational energy transfer. Unfortunately, for this system the fast charge transfer (Eq. (2)) precludes detailed analysis of the $v=2 \rightarrow v=0$ versus $v=2 \rightarrow v=1$ components in vibrational deactivation (Eq. (1)).

\subsection{Vibrational specificity in chemical reactions}

A dramatic enhancement in charge-transfer rates was observed by vibrational excitation of the $\mathrm{N}_{2}{ }^{+}$ion in the $\mathrm{N}_{2}{ }^{+}(v=0-4)+\mathrm{Kr}$ reactions at thermal energy ${ }^{17)}$; the rate constant increases by a factor of $\approx 80$ in going from $v=0$ to $v=4$. The observed increase is almost entirely due to enhanced charge transfer since the vibrational relaxation has been shown to be negligibly slow. ${ }^{16)}$ This is a particularly interesting system because charge transfer is highly exothermic for vibrational ground state $\mathrm{N}_{2}{ }^{+}$and yet the $v=0$ charge transfer is surprising slow $\left(k=1.0 \times 10^{-12} \mathrm{~cm}^{3} \mathrm{~s}^{-1}\right)$, occurring once in 800 collision events.

$$
\begin{aligned}
\mathrm{N}_{2}{ }^{+}(v=0)+\mathrm{Kr} & \rightarrow \mathrm{N}_{2}+\mathrm{Kr}^{+}\left({ }^{2} \mathrm{P}_{3 / 2}\right)+1.580 \mathrm{eV} \\
& \rightarrow \mathrm{N}_{2}+\mathrm{Kr}^{+}\left({ }^{2} \mathrm{P}_{1 / 2}\right)+0.915 \mathrm{eV}
\end{aligned}
$$

If energy mismatch is the problem, one may expect that charge transfer would be suppressed even more heavily for higher vibrational excitation in $\mathrm{N}_{2}{ }^{+}$as the apparent energy gap becomes greater. Based on this energy-gap argument, an early flow tube kinetics study ${ }^{31)}$ assigned the more reactive component to $\mathrm{N}_{2}{ }^{+}$ $(v=0)$ whereas the less reactive to $\mathrm{N}_{2}{ }^{+}(v>0)$. The state-specific detection with SIFT-LIF, however, unambiguously determined that the rates are enhanced by vibrational excitation of $\mathrm{N}_{2}^{+}(v)$.

The observed trend of vibrational enhancement qualitatively agrees with calculations using modified Rapp -Francis theory, ${ }^{17)}$ which formulates the state-selective reaction cross section $\sigma(v)$ as the product of an energyresonance term and the associated Franck-Condon factor $q_{v v^{\prime}}$, summed over all final $v^{\prime}$ states involved. This treatment has found considerable success in predicting state-selective reaction cross sections at high collision energies. ${ }^{32)}$ At $300 \mathrm{~K}\left(E_{\mathrm{cm}} \approx 0.04 \mathrm{eV}\right)$, however, the calculated rate constants for $\mathrm{N}_{2}^{+}(v)+\mathrm{Kr}$ are several orders of magnitude smaller than the experimental values. ${ }^{17)}$

The experimental result is quantitatively explained by a model ${ }^{17), 33)}$ that assumes, in reality, that the associated Franck-Condon factors for the $\mathrm{N}_{2}{ }^{+}(v)-\mathrm{N}_{2}\left(v^{\prime}\right)$ transitions are slightly perturbed during a close approach of the $\left(\mathrm{N}_{2}-\mathrm{Kr}\right)^{+}$pair (Fig. 2). When the ion and neutral approach at slow velocity, the wave functions for $\mathrm{N}_{2}{ }^{+}$and $\mathrm{N}_{2}$ would have sufficient time to adjust to each other. ${ }^{11)}$ To model such distortion of Franck-

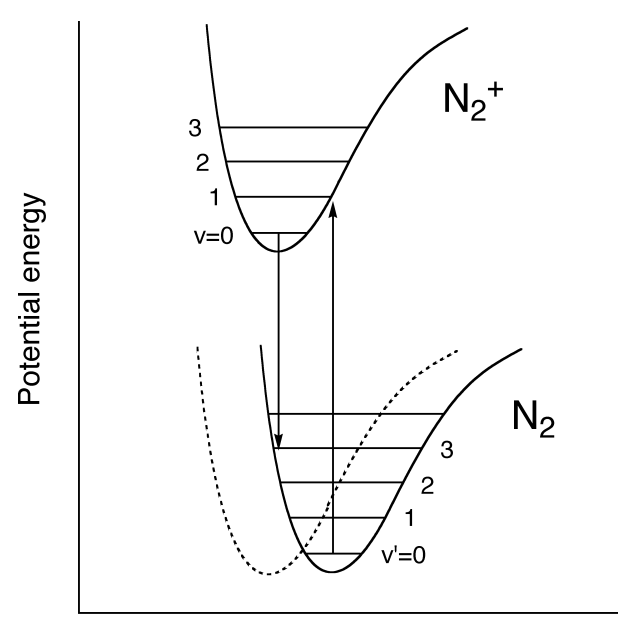

Internuclear distance

Fig. 2. Schematic energy diagram for $\mathrm{N}_{2}$ and $\mathrm{N}_{2}{ }^{+}$ representing the "distorted Franck-Condon" model. The wave functions and associated Franck-Condon factors are perturbed by the close approach of a reactant. In this example the $\mathrm{N}_{2}$ wave function is simply shifted from the equilibrium internuclear distance, shown by the dotted line. The displacement has been exaggerated for the sake of presentation. The Franck-Condon overlaps are consequently altered. 
Condon factors within the transient $\left(\mathrm{N}_{2}-\mathrm{Kr}\right)^{+}$pair, the vibrational wave functions of $\mathrm{N}_{2}{ }^{+}$and $\mathrm{N}_{2}$ are displaced relative to each other. In this instance the $\mathrm{N}_{2}$ wave function is simply shifted from the equilibrium internuclear distance $\left(r_{\mathrm{e}}\right)$ of $1.0977 \AA$ by a short displacement of $d=0.02 \AA$ (Eq. (6)), ${ }^{17), 33)}$ while the $\mathrm{N}_{2}{ }^{+}$wave function is fixed at $r_{\mathrm{e}}=1.1164 \AA$. The Franck-Condon overlap is consequently altered.

$$
q_{v, v^{\prime}}=\left|\int \Psi_{\mathrm{N}_{2}^{+}}(r) \Psi_{\mathrm{N}_{2}}(r+d) \mathrm{d} r\right|^{2}
$$

The rate constants for $\mathrm{N}_{2}{ }^{+}(v)+\mathrm{Kr}$ were calculated from the most energy-resonant product channels $(\Delta E<100$ $\mathrm{meV})$, in this case those producing $\mathrm{N}_{2}(v+3)+\operatorname{Kr}^{+}\left({ }^{2} \mathrm{P}_{1 / 2}\right)$ (Eqs. (7) - (11)). This is a reasonable approximation in the spirit of Rapp-Francis theory, as discussed in Kato et al., ${ }^{17)}$ that charge-transfer cross sections become extremely sensitive to the energy gap at low collision energies.

$$
\begin{aligned}
& \mathrm{N}_{2}^{+}(v=0)+\mathrm{Kr} \rightarrow \\
& \mathrm{N}_{2}\left(v^{\prime}=3\right)+\mathrm{Kr}^{+}\left({ }^{2} \mathrm{P}_{1 / 2}\right)+59 \mathrm{meV} \\
& \mathrm{N}_{2}{ }^{+}(v=1)+\mathrm{Kr} \rightarrow \\
& \mathrm{N}_{2}\left(v^{\prime}=4\right)+\mathrm{Kr}^{+}\left({ }^{2} \mathrm{P}_{1 / 2}\right)+51 \mathrm{meV} \\
& \mathrm{N}_{2}{ }^{+}(v=2)+\mathrm{Kr} \rightarrow \\
& \mathrm{N}_{2}\left(v^{\prime}=5\right)+\mathrm{Kr}^{+}\left({ }^{2} \mathrm{P}_{1 / 2}\right)+41 \mathrm{meV} \\
& \mathrm{N}_{2}^{+}(v=3)+\mathrm{Kr} \rightarrow \\
& \mathrm{N}_{2}\left(v^{\prime}=6\right)+\mathrm{Kr}^{+}\left({ }^{2} \mathrm{P}_{1 / 2}\right)+32 \mathrm{meV} \\
& \mathrm{N}_{2}{ }^{+}(v=4)+\mathrm{Kr} \rightarrow \\
& \mathrm{N}_{2}\left(v^{\prime}=7\right)+\mathrm{Kr}^{+}\left({ }^{2} \mathrm{P}_{1 / 2}\right)+22 \mathrm{meV}
\end{aligned}
$$

The "distorted Franck-Condon" model was originally developed by Lipeles to explain anomalous product vibrational state distributions in slow ion-molecule collisions. $^{34)}$ We extended his approach to predict stateselective reaction cross sections at low $E_{\mathrm{cm}}$, where the Rapp-Francis treatment starts to fail. We also applied this model to successfully reproduce the $\mathrm{N}_{2}{ }^{+}(v=1-4)$ + Ar charge transfer rates. ${ }^{33)}$ The measured rate constants are nearly unchanged over the exothermic channels $\mathrm{N}_{2}{ }^{+}(v=1-4)+\mathrm{Ar} \rightarrow \mathrm{N}_{2}+\mathrm{Ar}^{+}$, a result unexplained by standard Franck-Condon arguments.

Vibrational specificity in reactions of $\mathrm{N}_{2}{ }^{+}(v)$ with diatomic reagents was also studied. Upon vibrational excitation, the charge-transfer component for the $\mathrm{N}_{2}{ }^{+}$ $(v)+\mathrm{CO}$ reaction ${ }^{22)}$ increases considerably whereas the $\mathrm{N}_{2}{ }^{+}(v)+\mathrm{NO}^{22)}$ and $\mathrm{N}_{2}{ }^{+}(v)+\mathrm{O}_{2}$ charge transfer rates ${ }^{18), 21)}$ show only moderate increases. These observations are intriguing since, for both the $\mathrm{NO}$ and $\mathrm{O}_{2}$ reactions, electronically excited states of the ion products become energetically accessible, i.e., $\mathrm{NO}^{+}\left(a^{3} \Sigma^{+}\right)$from $\mathrm{N}_{2}{ }^{+}(v \geq 1)$ and $\mathrm{O}_{2}{ }^{+}\left(a^{4} \Pi_{\mathrm{u}}\right)$ from $\mathrm{N}_{2}{ }^{+}(v \geq 2)$. In contrast, for the $\mathrm{N}_{2}{ }^{+}$ $(v)+\mathrm{CO}$ reaction, the lowest electronically excited product ion state $\mathrm{CO}^{+}\left(A^{2} \Pi\right)$ is not thermodynamically accessible. The moderate increase in the $\mathrm{N}_{2}{ }^{+}(v \geq 2)+\mathrm{O}_{2}$ charge transfer rate has been explained theoretically by state coupling via the quartet $\left[\mathrm{N}_{2} \cdot \mathrm{O}_{2}\right]^{+}$complex ${ }^{26)}$ and also by wavepacket dynamics. ${ }^{35)}$ A guided ion beam experiment reported an anomalously small charge-transfer reactivity for $\mathrm{N}_{2}{ }^{+}(v=1)+\mathrm{O}_{2} \cdot{ }^{36)}$ However, such a decrease is not observed in other studies using mass spectrometric kinetics (reviewed in Kato et al. ${ }^{18), 21)}$ ) and photoelectron-photoion coincidence, ${ }^{37)}$ and the discrepancy remains unresolved.

We also studied vibrational specificity in the hydrogen-atom transfer reaction $\mathrm{N}_{2}{ }^{+}(v)+\mathrm{H}_{2} \rightarrow \mathrm{N}_{2} \mathrm{H}^{+}+\mathrm{H}^{23)}$ The reaction rates are large and nearly unchanged with respect to the reactant vibrational excitation. The fact that the rates significantly exceed the Langevin collision limit suggests that the reaction proceeds via initial charge transfer at large intermolecular distances producing $\mathrm{N}_{2}+\mathrm{H}_{2}{ }^{+}$, followed by proton transfer at a shorter range. The Langevin rate is greater for $\mathrm{N}_{2}+\mathrm{H}_{2}{ }^{+}$than for $\mathrm{N}_{2}{ }^{+}+\mathrm{H}_{2}$. Vibrational specificity may well be smeared out during these sequential processes in a single collision event.

\section{Dynamics of Organic Ion-Molecule Reactions}

\subsection{Nucleophilic substitution $\left(\mathrm{S}_{\mathrm{N}} 2\right)$ reactions}

Nucleophilic substitution is one of the most fundamental chemical transformations in solution, and there have been numerous gas phase studies as well. ${ }^{38)}$ In the absence of solvent molecules, gas phase $\mathrm{S}_{\mathrm{N}} 2$ studies reveal intrinsic features of this reaction (mechanism and energetics, transition state structure, energy transfer/randomization, product dynamics) as well as provide key calibration for theory. This part discusses mechanisms and dynamic effects in $\mathrm{S}_{\mathrm{N}} 2$ reactions in some detail to provide a background for later sections.

$\mathrm{S}_{\mathrm{N}} 2$ reactions of methyl halides with halide anions, $\mathrm{X}^{-}+\mathrm{CH}_{3} \mathrm{Y} \rightarrow \mathrm{CH}_{3} \mathrm{X}+\mathrm{Y}^{-}$, represent the simplest system in this category. The SIFT technique was used to detect the occurrence of a slow, symmetric $\mathrm{S}_{\mathrm{N}} 2$ reaction $(\mathrm{X}=\mathrm{Y}=\mathrm{Cl})$ in the gas phase. ${ }^{39)}$

$$
{ }^{37} \mathrm{Cl}^{-}+\mathrm{CH}_{3}{ }^{35} \mathrm{Cl} \rightarrow \mathrm{CH}_{3}{ }^{37} \mathrm{Cl}+{ }^{35} \mathrm{Cl}^{-}
$$

The reaction is extremely slow at room temperature with the measured rate coefficient of $k \approx 3.5 \times 10^{-14} \mathrm{~cm}^{3}$ $\mathrm{s}^{-1}$ (one reaction in $\approx 50000$ collisions). The remarkable slowness of the thermoneutral $\mathrm{S}_{\mathrm{N}} 2$ reaction is in sharp contrast to the general rapidity of proton-transfer $(\mathrm{PT})$ reactions, which occur at nearly every collision for many systems that are thermoneutral or nearthermoneutral. ${ }^{40)}$

Figure 3 schematically shows potential energy curves for $\mathrm{S}_{\mathrm{N}} 2$ and PT processes, for which the reactions are assumed to be similarly exothermic by $\Delta E_{\text {rxn }}$. The transition state (TS) for proton transfer is located deep in the ion-molecule attractive potential well (Fig. 3a); the TS barrier is as low as a few kcal $\mathrm{mol}^{-1}$ or virtually nonexistent for typical PT systems, e.g., those involving oxo acids. ${ }^{41)}$ In contrast, for $\mathrm{S}_{\mathrm{N}} 2$ transformation involving Walden inversion (Fig. 3b), the central energy barrier $\left(\Delta E^{\neq}\right.$cent $)$is considerably high and comparable with the well depth (complexation energy $\left.\Delta E_{\text {comp }} \approx 10-25 \mathrm{kcal} \mathrm{mol}^{-1}\right) .{ }^{42)}$ The double-well scheme was first proposed by Olmstead and Brauman ${ }^{43)}$ for solvent-free $\mathrm{S}_{\mathrm{N}} 2$ reactions in the gas phase. Even when the transition state is somewhat lower in energy than the reactants (i.e., the overall energy barrier $\Delta E^{\neq}{ }_{\text {ovr }}<0$ ), back-dissociation of the reactant complex $\left[\mathrm{X}^{-} \cdots \mathrm{CH}_{3} \mathrm{Y}\right]$ is favored over Walden inversion to form the product complex $\left[\mathrm{XCH}_{3} \cdots \mathrm{Y}^{-}\right]$, based on a simple density-ofstates argument. The reaction efficiency can thus be 


\section{(a) Proton transfer}

$$
\mathrm{B}^{-}+\mathrm{HA} \rightarrow\left[\mathrm{B}^{---\mathrm{H}^{--}-\mathrm{A}}\right]_{T S}^{-} \rightarrow \mathrm{BH}+\mathrm{A}^{-}
$$

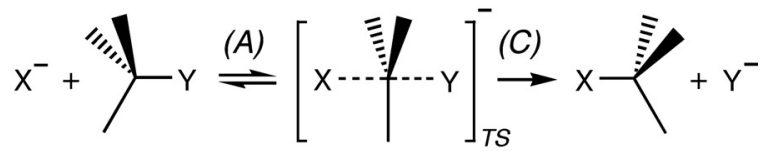

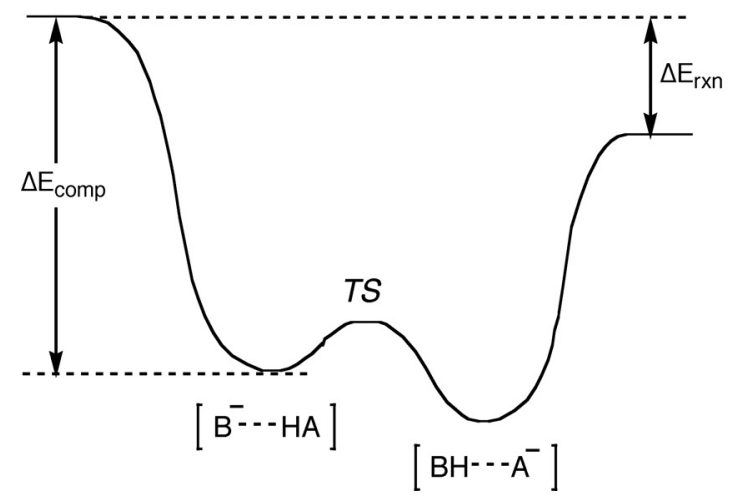

(B)

(b) Nucleophilic substitution $\left(\mathrm{S}_{\mathrm{N}} 2\right)$

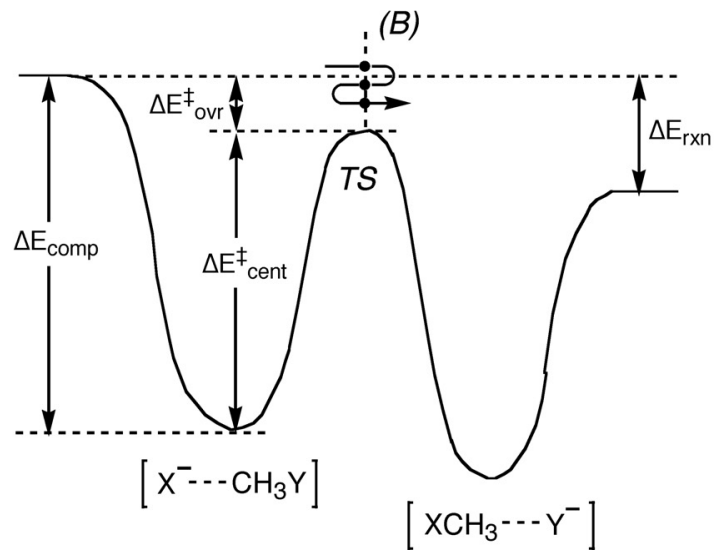

Fig. 3. Schematic potential energy curves for (a) proton transfer reaction of base anion $\mathrm{B}^{-}$with acid HA, and (b) $\mathrm{S}_{\mathrm{N}} 2$ reaction of nucleophile $\mathrm{X}^{-}$with methyl halide $\mathrm{CH}_{3} \mathrm{Y}$, in the gas phase. The diagram (a) represents typical protontransfer processes involving oxo- and sulfo- acids. Zero-point energies have been omitted for simplicity.

considerably small.

The slow $\mathrm{S}_{\mathrm{N}} 2$ reaction (Eq. (12)) is dramatically enhanced as the ion-molecule collision energy is increased in the SIFT-drift field, ${ }^{39)}$ stimulating fundamental interest in the mechanism of gas phase nucleophilic substitution. A high-level ab initio theoretical study ${ }^{44)}$ suggested this behavior as evidence for the occurrence of a novel "front-side" $\mathrm{S}_{\mathrm{N}} 2$ mechanism, in which ${ }^{37} \mathrm{Cl}^{-}$ approaches the carbon atom from the same side as ${ }^{35} \mathrm{Cl}^{-}$leaves (Fig. 4b). At the $\mathrm{G} 2(+)$ level of theory ${ }^{44)}$ the energy threshold for the front-side attack was predicted to be $46.3 \mathrm{kcal} \mathrm{mol}^{-1}$. However, guided ion beam experiments under single collision conditions ${ }^{45}$ ) measured the threshold for the translational activation to be $11 \pm 4 \mathrm{kcal} \mathrm{mol}^{-1}$, significantly lower than the theoretical front-side threshold. Therefore, the frontside mechanism must not be reasonable. The observed enhancement can then be attributed to translational activation of the conventional back-side $\mathrm{S}_{\mathrm{N}} 2$ reaction (Fig. 4a), which must be dynamically restricted (see below). A more recent, $a b$ initio direct dynamics trajectory simulation supports the back-side $\mathrm{S}_{\mathrm{N}} 2$ mechanism $^{46)}$; substantial vibrational excitation in the $\mathrm{CH}_{3} \mathrm{Cl}$ reactant is required to access the front-side transition state, and even so, the front-side pathway will still be dominated by the back-side reaction.

For the thermoneutral $\mathrm{Cl}^{-}+\mathrm{CH}_{3} \mathrm{Cl}$ system, the transition state for the back-side $\mathrm{S}_{\mathrm{N}} 2$ reaction is actually calculated to be slightly higher in energy than the reactants at the $\mathrm{G} 2(+)$ level of theory $\left(\Delta E^{\neq}{ }_{\text {ovr }}=+2.7\right.$ $\left.\mathrm{kcal} \mathrm{mol}{ }^{-1}\right){ }^{47)}$ This is consistent with the fact that the reaction is very slow at $300 \mathrm{~K}$ and accelerated by translational excitation. However, why is the translational threshold $\left(\approx 11 \mathrm{kcal} \mathrm{mol}^{-1}\right)^{45)}$ significantly greater than the overall energy barrier? As it turned out, the "simple" $\mathrm{S}_{\mathrm{N}} 2$ reaction (Eq. (12)) is far from simple. The $\mathrm{Cl}^{-}+\mathrm{CH}_{3} \mathrm{Cl}$ reaction is a member of methyl halide $\mathrm{S}_{\mathrm{N}} 2$ (a) Back-side $\mathrm{S}_{\mathrm{N}} 2$

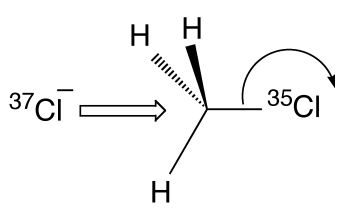

(b) "Front-side" $\mathrm{S}_{\mathrm{N}} 2$

(c) $\mathrm{S}_{\mathrm{N}} 2$ at nitrogen

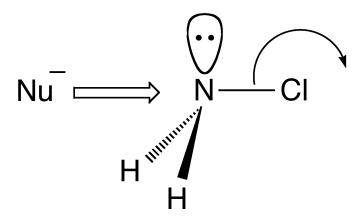

Fig. 4. Various types of nucleophilic substitution reactions. (a) conventional back-side $\mathrm{S}_{\mathrm{N}} 2$, (b) "front-side" $\mathrm{S}_{\mathrm{N}} 2$, and (c) $\mathrm{S}_{\mathrm{N}} 2$ at nitrogen.

reactions that display nonstatistical kinetics and dynamics (see Sun, Song, and Hase ${ }^{48), 49)}$ and references therein). The short lifetime of the initial $\left[\mathrm{Cl}^{-} \cdots \mathrm{CH}_{3} \mathrm{Cl}\right]$ complex ( $\tau \approx 4 \mathrm{ps}$ ) hampers efficient coupling of the $\mathrm{Cl}^{-}$ $-\mathrm{CH}_{3} \mathrm{Cl}$ intermolecular mode (i.e., translational energy) and $\mathrm{CH}_{3} \mathrm{Cl}$ intramolecular mode, resulting in the failure to utilize the translational energy to surmount the central $\mathrm{S}_{\mathrm{N}} 2$ barrier. ${ }^{48}$ ) Orientational and angularmomentum constraints are also suggested to explain the inefficient translational activation observed.45), 50) While statistical behaviors have been demonstrated for large $\mathrm{S}_{\mathrm{N}} 2$ systems with complex lifetimes $\tau>10 \mathrm{~ns},{ }^{51)}$ $\mathrm{S}_{\mathrm{N}} 2$ reactions of methyl halides are nonstatistical in many cases primarily because of the inefficient intramolecular vibrational relaxation (IVR) within the complex lifetimes of $\sim 10$ ps or shorter. Such incom- 
plete energy randomization violates a basic assumption in statistical rate theories, e.g., canonical, transition state theory (TST) and microcanonical, RiceRamsperger-Kassel-Marcus (RRKM) theory. ${ }^{52), 53)}$

In discussing "nonstatistical" behaviors, it should be clarified in what region of the potential energy surface IVR is impeded and how it affects the experimental observables. Statistical rate theories assume complete randomization of energy (i.e., statistical population of phase space) all the way from the reactants via the initial complex $u p$ to the transition state ( $A$ in Fig. $3 \mathrm{~b}$ ). Thus, nonstatistical behaviors show up as deviations in rate kinetics (e.g., kinetic isotope effects, see below) and as differential effects of reactant excitation (translation, rotation, vibration) on the reaction rates. ${ }^{54), 55)}$ When energy randomization is hampered after the transition state all the way down to the products $(C$ in Fig. 3b), nonstatistical dynamics are observed as anomalous product energy distributions, ${ }^{56), 57)}$ while the rate kinetics may behave normally. It has also been predicted that the $\mathrm{S}_{\mathrm{N}} 2$ reaction of $\mathrm{HO}^{-}+\mathrm{CH}_{3} \mathrm{~F}$ avoids the deep potential energy minimum in the exit channel ${ }^{49)}$; it is caused by inefficient IVR in the post TS region within the time scale of the product complex dissociation.

A more subtle case is inefficient translation-tovibration $(\mathrm{T}-\mathrm{V})$ energy coupling in the TS region $(B$ in Fig. 3b). As shown by simulations for the $\mathrm{Cl}^{-}+\mathrm{CH}_{3} \mathrm{Br}$ and $\mathrm{Cl}^{-}+\mathrm{CH}_{3} \mathrm{Cl}$ systems, ${ }^{48), 57)}$ this traps the complex in the central barrier region and allows multiple TS recrossings to occur, another violation of a basic assumption in statistical rate theories. ${ }^{52), 53)}$ The reaction probability per ion-molecule collision will consequently decrease. Direct techniques such as transition-state spectroscopy may be used to probe the transient species trapped in this region. Recently, femtosecond time-resolved photoelectron spectroscopy has been employed $^{58)}$ to monitor the dissociation dynamics of an energized $\mathrm{S}_{\mathrm{N}} 2$ complex I ${ }^{-} \cdot \mathrm{CH}_{3} \mathrm{I}$. Because of the experimental constraints ${ }^{58)}$ the complex cannot have sufficient energy to surmount the $\mathrm{S}_{\mathrm{N}} 2$ barrier. In this symmetric system the $\mathrm{S}_{\mathrm{N}} 2$ process is not distinguishable from complex dissociation, either. Nonetheless, a future breakthrough may be expected with these approaches.

\subsection{Kinetic isotope effects in $\mathrm{S}_{\mathrm{N}} 2$ reactions}

Kinetic isotope effects (KIEs) ${ }^{59)}$ have been used as a somewhat indirect but sensitive probe for the mechanism and transition state structure of $\mathrm{S}_{\mathrm{N}} 2$ reactions. For methyl halides reacting with nucleophile anions $\left(\mathrm{Nu}^{-}\right)$, the secondary $\alpha$-deuterium KIE is defined as the ratio of the rate constants for unlabeled $\left(k_{\mathrm{H}}\right)$ to deuterated $\left(k_{\mathrm{D}}\right) \mathrm{S}_{\mathrm{N}} 2$ substrates $\left(K I E \equiv k_{\mathrm{H}} / k_{\mathrm{D}}\right)$.

$$
\begin{aligned}
& \mathrm{CH}_{3} \mathrm{X}+\mathrm{Nu}^{-} \stackrel{k_{\mathrm{H}}}{\longrightarrow} \mathrm{CH}_{3} \mathrm{Nu}+\mathrm{X}^{-} \\
& \mathrm{CD}_{3} \mathrm{X}+\mathrm{Nu}^{-} \stackrel{k_{\mathrm{D}}}{\longrightarrow} \mathrm{CD}_{3} \mathrm{Nu}+\mathrm{X}^{-}
\end{aligned}
$$

The inverse $\operatorname{KIE}\left(k_{\mathrm{H}} / k_{\mathrm{D}}<1\right)$ is generally consistent with a tight $\mathrm{S}_{\mathrm{N}} 2$ transition state, for which the overall energy barrier $\Delta E^{\neq}$ovr is slightly higher for the protonated reactant than for the deuterated reactant. ${ }^{52)} \mathrm{A}$ working hypothesis in quantitatively discussing KIEs is that the reaction is statistical, i.e., there is complete energy randomization all the way from the reactants up to the transition state. The assumption allows for prediction of KIEs based on conventional TST and the associated partition function relationship between the reactants and transition state. Deviations from statistical dynamics are noticed as the difference between experimental and theoretical KIEs, either in the magnitude for a specific reaction, or in the trend for a series of systematic $\mathrm{S}_{\mathrm{N}} 2$ reactions.

To illustrate the latter, Poirier et al. ${ }^{60)}$ devised a transition-state "looseness" parameter, $R_{\mathrm{TS}}$ (Fig. 5a). For a given $\mathrm{S}_{\mathrm{N}} 2$ substrate reacting with different nucleophiles, they demonstrated computationally that KIE values are greater for nucleophiles that have larger $R_{\mathrm{TS}}$. As $R_{\mathrm{TS}}$ increases and the transition state becomes looser, more "space" becomes available around the $\mathrm{C}_{\alpha}-$ $\mathrm{H}(\mathrm{D})$ bonds at the transition state. Thus, at the transition state, the out-of-plane $\mathrm{C}-\mathrm{H}$ bending frequencies are lowered more dramatically than are the corresponding $\mathrm{C}-\mathrm{D}$ frequencies, leading to an increase in KIEs from inverse toward normal $\left(k_{\mathrm{H}} / k_{\mathrm{D}}>1\right)$. This effect is contrasted by that of methyl $\mathrm{C}-\mathrm{H}$ stretching frequencies, which are the major contributor to the overall inverse KIE but are nearly unchanged across different nucleophiles. ${ }^{60)}$

Under well-defined thermal energy conditions with the SIFT technique, we determined $\alpha$-deuterium KIEs ${ }^{61)}$ for $\mathrm{S}_{\mathrm{N}} 2$ reactions of methyl halides $\mathrm{CH}_{3} \mathrm{Cl}, \mathrm{CH}_{3} \mathrm{Br}$, and $\mathrm{CH}_{3} \mathrm{I}$ and compared them with theoretical predictions (Kato et al. ${ }^{61)}$ and references cited therein). Experimental KIEs for methyl chloride are found to increase from inverse toward normal as $R_{\mathrm{TS}}$ increases (Fig. 6), in excellent agreement with TST predictions in both the trend and magnitude. This suggests that $\mathrm{S}_{\mathrm{N}} 2$ reactions of $\mathrm{CH}_{3} \mathrm{Cl}$ proceed statistically. There is a caveat, however, that consistent KIEs do not necessarily warrant (a)

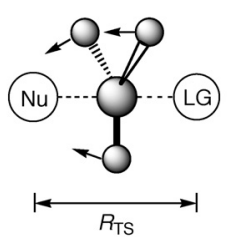

(c)

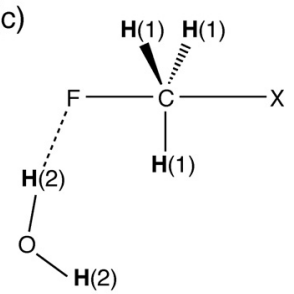

(b)

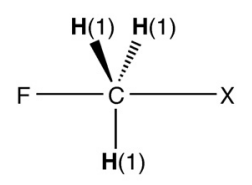

(d)

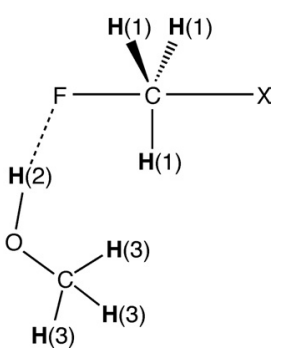

Fig. 5. (a) The transition-state "looseness" parameter $\left(R_{\mathrm{TS}}\right)$, defined as the sum of the lengths of the nucleophile atom $(\mathrm{Nu})$-methyl carbon $\left(\mathrm{C}_{\alpha}\right)$ and methyl carbon-leaving group atom (LG). Schematically shown is the out-of-plane $\mathrm{C}_{\alpha}-\mathrm{H}$ (D) umbrella mode at the transition state. Schematic $\mathrm{S}_{\mathrm{N}} 2$ transition states for methyl halides: (b) $\mathrm{F}^{-}+\mathrm{CH}_{3} \mathrm{X}$, (c) $\mathrm{F}^{-}\left(\mathrm{H}_{2} \mathrm{O}\right)+\mathrm{CH}_{3} \mathrm{X}$, and (d) $\mathrm{F}^{-}\left(\mathrm{CH}_{3} \mathrm{OH}\right)+\mathrm{CH}_{3} \mathrm{X}$. The reagents can be specifically deuterated at the methyl halide $(\mathrm{H}$ (1)), the hydroxyl group $(\mathrm{H}(2))$, or the methyl group in methanol $(\mathrm{H}(3))$. 


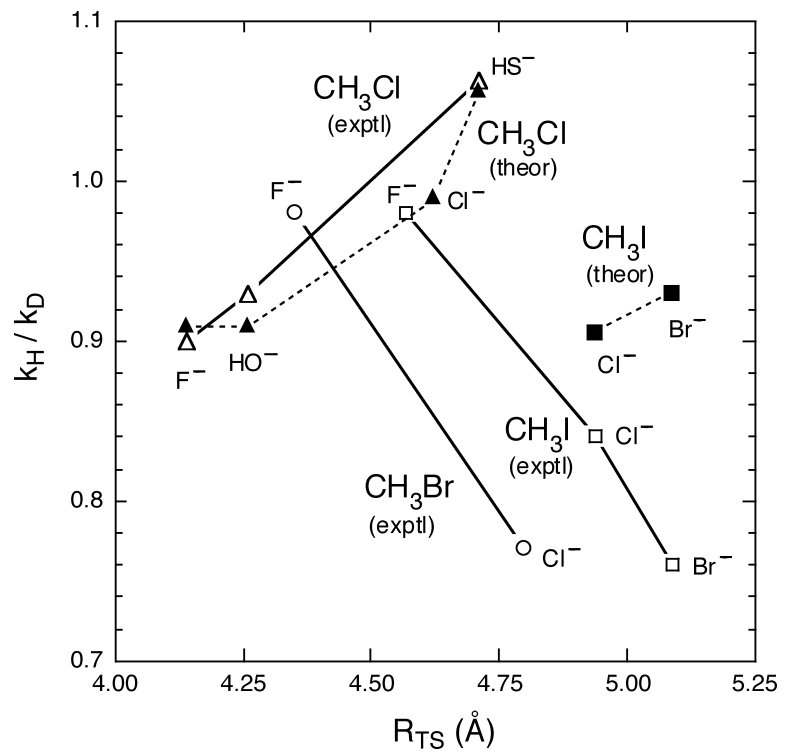

Fig. 6. Experimental (open symbols) and theoretical (closed symbols) KIEs ( $\left.\equiv k_{\mathrm{H}} / k_{\mathrm{D}}\right)$ as a function of the transition-state looseness parameter $\left(R_{\mathrm{TS}}\right)$ for the $\mathrm{S}_{\mathrm{N}} 2$ reactions of methyl halides with a variety of nucleophiles in the gas phase. Data taken from Kato et al. ${ }^{61)}$ The lines are intended only to guide the eye.

statistical dynamics in every aspect of the reaction. Among the methyl chloride series, the $\mathrm{CH}_{3} \mathrm{Cl}+\mathrm{F}^{-}$reaction shows a nonstatistical behavior such that the rate is independent of the reactant internal energy. ${ }^{54)} \mathrm{A}$ direct mechanism without formation of a long-lived complex has been suggested from the observed deviation of reaction cross sections at high collision energies $^{62)}$ and by ab initio direct dynamics calculations (collision energy not specified). ${ }^{63)}$ The $\mathrm{CH}_{3} \mathrm{Cl}+\mathrm{Cl}^{-}$reaction proceeds via a similar direct (nonstatistical) mechanism associated with favored reactant orientation, ${ }^{45), 48), 50)}$ and nonstatistical barrier-recrossing is also predicted. ${ }^{48)}$ As such, KIEs are only sensitive to nonstatistical attributes that do not cancel in the rate ratio $k_{\mathrm{H}} / k_{\mathrm{D}}$. By definition, they are also blind to dynamics after the transition state region.

For $\mathrm{S}_{\mathrm{N}} 2$ reactions of heavier methyl halides, observed $\alpha$-deuterium KIEs are distinctly anomalous. ${ }^{61)}$ Experimental KIE values for $\mathrm{CH}_{3} \mathrm{Br}$ and $\mathrm{CH}_{3} \mathrm{I}$ decrease (i.e., become more inverse) as $R_{\mathrm{TS}}$ increases (Fig. 6). Interestingly, computed partition functions for $\mathrm{CH}_{3} \mathrm{I}$ still predict a normal, positive correlation between the KIE value and the looseness parameter. Although magnitudes of computational KIE values for individual reactions may vary depending on the theoretical methods employed, ${ }^{64), 65)}$ the opposite trend between experiments and statistical theory clearly indicates that $\mathrm{S}_{\mathrm{N}} 2$ dynamics of the heavier methyl halides is nonstatistical. Other kinetics observations also suggest incomplete IVR for these systems. ${ }^{54)}$ The transition-state looseness model has been modified by incorporating steric effects in more refined manners, e.g., consideration of atomic radii for the nucleophile and leaving groups $^{66)}$ and three dimensional "crowdedness" imposed by polyatomic nucleophiles. ${ }^{67)}$ However, the observed discrepancy in the KIE trend cannot be ex- plained by these adjustments.

SIFT studies have been extended to microsolvated $\mathrm{S}_{\mathrm{N}} 2$ reactions, which have attracted considerable attention recently. ${ }^{38)}$, 68), 69) Reactions of $\mathrm{F}^{-}\left(\mathrm{H}_{2} \mathrm{O}\right)+\mathrm{CH}_{3} \mathrm{X}(\mathrm{X}$ $=\mathrm{Cl}, \mathrm{Br}, \mathrm{I})$ and their deuterated analogs display substantial inverse KIEs by deuteration of the water solvent as well as of the methyl group in methyl halides. ${ }^{70)}$ For unlabeled reactants, the reaction proceeds via two channels (Eqs. (15) and (16)).

$$
\begin{aligned}
\mathrm{F}^{-}\left(\mathrm{H}_{2} \mathrm{O}\right)+\mathrm{CH}_{3} \mathrm{X} & \rightarrow \mathrm{X}^{-}+\mathrm{CH}_{3} \mathrm{~F}+\mathrm{H}_{2} \mathrm{O} \\
& \rightarrow \mathrm{X}^{-}\left(\mathrm{H}_{2} \mathrm{O}\right)+\mathrm{CH}_{3} \mathrm{~F}
\end{aligned}
$$

The measured KIE values are in excellent agreement with TST predictions, which have been made for $\mathrm{F}^{-}$ $\left.\left(\mathrm{H}_{2} \mathrm{O}\right)+\mathrm{CH}_{3} \mathrm{Cl}^{71}\right)$ This may not be surprising since IVR is expected to be very efficient for these large systems. The product branching, however, shows intriguing dynamical effects. For this reaction, the measured product yields for Eq. (15) (including potential formation of $\left.\mathrm{CH}_{3} \mathrm{~F}\left(\mathrm{H}_{2} \mathrm{O}\right)+\mathrm{Cl}^{-}\right)^{72)}$ and Eq. (16) are approximately $70 \%$ and $30 \%$, respectively, despite the fact that the latter is significantly more favorable energetically. Tachikawa performed direct $a b$ initio dynamics calculations at higher collision energies $\left(E_{\mathrm{cm}}=4-25 \mathrm{kcal} \mathrm{mol}^{-1}\right)$ and predicted that formation of $\mathrm{Cl}^{-}\left(\mathrm{H}_{2} \mathrm{O}\right)$ (Eq. (16)) is inefficient because of a geometrical/dynamical constraint after the $\mathrm{S}_{\mathrm{N}} 2$ transformation. ${ }^{72), 73)}$

While the measured isotope effects are consistent with TST, a factor analysis dissecting the overall KIEs provides useful clues to the structure and molecular interaction at the $\mathrm{S}_{\mathrm{N}} 2$ transition state. ${ }^{67), 71)}$ The transition-state structure for the $\mathrm{F}^{-}\left(\mathrm{H}_{2} \mathrm{O}\right)+\mathrm{CH}_{3} \mathrm{X}$ reactions is schematically shown in Fig. 5c, in comparison to the bare ion system $\mathrm{F}^{-}+\mathrm{CH}_{3} \mathrm{X}$ (Fig. $5 \mathrm{~b}$ ). In addition to the inverse methyl halide KIE as explained by a mechanism similar to the bare ion case, the strong inverse KIE upon deuteration of the water solvent is due primarily to the stretching vibration between the oxygen atom and the hydrogen atom $\mathrm{H}(2)$ that is hydrogen bonded to fluorine (Fig. 5c). ${ }^{67)}$, 71) The $\mathrm{O}-\mathrm{H}(2)$ stretching frequency increases at the transition state compared to that in free $\mathrm{F}^{-}\left(\mathrm{H}_{2} \mathrm{O}\right)$, giving rise to the strong inverse isotope effect. ${ }^{67)}, 71$ )

We further studied KIEs for the $\mathrm{S}_{\mathrm{N}} 2$ reactions of $\mathrm{CH}_{3} \mathrm{X}$ with $\mathrm{F}^{-}$(methanol), $\mathrm{F}^{-}$(isopropyl alcohol), and $\mathrm{F}^{-}$ (hydrogen fluoride). ${ }^{74)}$ Substantial inverse KIEs were observed in all these reactions by deuteration of the hydroxyl group of the solvent (or deuteration of hydrogen fluoride). This is totally consistent with the $\mathrm{F}^{-}$ $\left(\mathrm{H}_{2} \mathrm{O}\right)+\mathrm{CH}_{3} \mathrm{X}$ results ${ }^{70}$; the kinetic isotope effects could similarly arise from the increase in the $\mathrm{O}-\mathrm{H}(2)$ stretching frequency at the transition state, as schematically illustrated for $\mathrm{F}^{-}$(methanol) $+\mathrm{CH}_{3} \mathrm{X}$ (Fig. 5d) although no structure and vibrational data are available. The inverse $\mathrm{KIE}$ observed for $\mathrm{F}^{-}(\mathrm{HF})$ versus $\mathrm{F}^{-}(\mathrm{DF})$ is explained in essentially the same manner. Moderate inverse KIEs were also measured for deuteration of the remote methyl group in methanol $(\mathrm{H}(3)$ in Fig. $5 \mathrm{~d})$. At the transition state for $\mathrm{F}^{-}\left(\mathrm{H}_{2} \mathrm{O}\right)+\mathrm{CH}_{3} \mathrm{Cl}$ (Fig. 5c) the terminal $\mathrm{O}-\mathrm{H}(2)$ bond interacts with the methyl group in $\mathrm{CH}_{3} \mathrm{Cl}$, giving rise to low-frequency fundamental modes and contributing to the inverse isotope effects 
observed. ${ }^{71)}$ We speculate that a similar mechanism is operative for the KIE upon the deuteration of $\mathrm{H}(3)$ in methanol. ${ }^{74)}$

\subsection{Hydrogen/deuterium exchange and scrambl-} ing

In highly exothermic ion-molecule reactions, lifetime of the intermediate complex can be substantially short that rapid complex dissociation may effectively compete with other chemical processes that are to occur therein. The reaction of $\mathrm{CH}_{3}{ }^{+}$with $\mathrm{CH}_{4}$ is exothermic by nearly $30 \mathrm{kcal} \mathrm{mol}^{-1}$ and produces the ethyl cation and $\mathrm{H}_{2}$ via the protonated ethane intermediate complex $\left[\mathrm{C}_{2} \mathrm{H}_{7}{ }^{+}\right]$. Hiraoka and Kebarle ${ }^{75)}$ found evidence for the existence of two structures for the $\mathrm{C}_{2} \mathrm{H}_{7}{ }^{+}$intermediate, both classical and non-classical. The SIFT technique was used to probe the hydrogen/deuterium (H/D) scrambling process during this reaction, ${ }^{76)}$ by starting from differently labeled reactants, e.g., $\mathrm{CD}_{3}{ }^{+}+\mathrm{CH}_{4}$ (Scheme 1), $\mathrm{CHD}_{2}{ }^{+}+\mathrm{CH}_{3} \mathrm{D}$, and $\mathrm{CH}_{3}{ }^{+}+\mathrm{CHD}_{3}$ and observing variations in the losses of $\mathrm{H}_{2}, \mathrm{HD}$, and $\mathrm{D}_{2}$. Isotope scrambling occurs via interconversion between the non-classical $\mathbf{1}$ and classical 2 structures. The reaction is all the way downhill relative to the reactants, since the relative energies of those intermediates as well as of the connecting transition states are significantly lower than the reactant energy by at least 20 kcal $\mathrm{mol}^{-1}$. ${ }^{75), 77)}$ If the complex is sufficiently longlived, equilibrium between the two structures is established and the losses of hydrogen will be statistical and the same, regardless of the different deuteration in the reactants. The experiments indicate that the scrambl-

$$
\begin{aligned}
& \mathrm{CD}_{3}^{+}+\mathrm{CH}_{4} \longrightarrow\left[\stackrel{\mathrm{H}}{\stackrel{\mathrm{H}}{-}} \mathrm{DH}_{3}\right]^{*} \\
& 1
\end{aligned}
$$

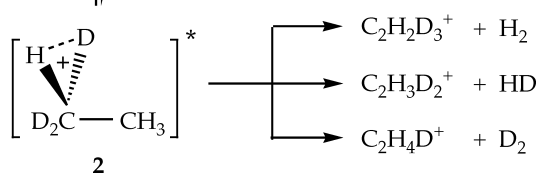

Scheme 1. H/D scrambling following reactive collision of $\mathrm{CD}_{3}{ }^{+}$and $\mathrm{CH}_{4}$. ing is incomplete and there are subtle differences among the individual reactant systems. This is caused by rapid dissociation of the complex; a modeling shows that approximately seven $\mathrm{H} / \mathrm{D}$ scramblings occur before the complex dissociates to products. ${ }^{76)}$

Hydrogen/deuterium exchange of ions with neutral reagents is a thermoneutral process besides the small isotope effects. In anion $\mathrm{H} / \mathrm{D}$ exchange, the reaction is considered to proceed via a key intermediate complex 3 , in which all hydrogen atoms are identical (Scheme 2). ${ }^{78)}$ Abstraction of a proton by $\mathrm{B}^{-}$followed by complex dissociation results in one H/D exchange. By repeating collisions with $\mathrm{BD}$, all hydrogen atoms in $\mathrm{AH}_{n}{ }^{-}$may exchange. We observed anomalous $\mathrm{H} / \mathrm{D}$ exchange for mono- and difluorophenyl anions reacting with $\mathrm{D}_{2} \mathrm{O}$ and $\mathrm{CH}_{3} \mathrm{OD} .{ }^{79}$ ) The observed behavior reveals the dynamical motion within the ion-dipole complex. For example in the $p$-difluorophenyl anion, all three hydrogen atoms are thermodynamically identical after intracomplex deuteron transfer to form $\mathbf{5}$ or $\mathbf{6}$, neglecting any small isotope effects (Scheme 3 ). Thus one would expect that the three hydrogen atoms in the anion will exchange with either reagent. In fact, $\mathrm{CH}_{3} \mathrm{OD}$ does exchange all three, but $\mathrm{D}_{2} \mathrm{O}$ exchanges only one. This indicates that $\mathrm{DO}^{-}$is trapped on one side of the benzene ring and cannot access the other side bearing two hydrogen atoms, whereas $\mathrm{CH}_{3} \mathrm{O}^{-}$can migrate freely within the complex.

The kinetically restricted exchange with $\mathrm{D}_{2} \mathrm{O}$ can be understood in terms of the calculated energy diagram. ${ }^{79)}$ Ab initio computations indicate that the $\mathrm{DO}^{-}$species can migrate along the side of the benzene ring where it is formed (5); however, it cannot migrate across the barrier caused by the fluorine atoms or by

$$
\mathrm{AH}_{\mathrm{n}}^{-}+\mathrm{BD} \longrightarrow\left[\mathrm{AH}_{\mathrm{n}} \mathrm{D} \cdots \mathrm{B}^{-}\right] \rightleftharpoons\left[\mathrm{AH}_{\mathrm{n}-1} \mathrm{D}^{-} \cdots \mathrm{BH}\right]
$$

Scheme 2. $\mathrm{H} / \mathrm{D}$ exchange reaction of an anion $\mathrm{AH}_{n}{ }^{-}$ with a deuterated neutral reagent $\mathrm{BD}$.

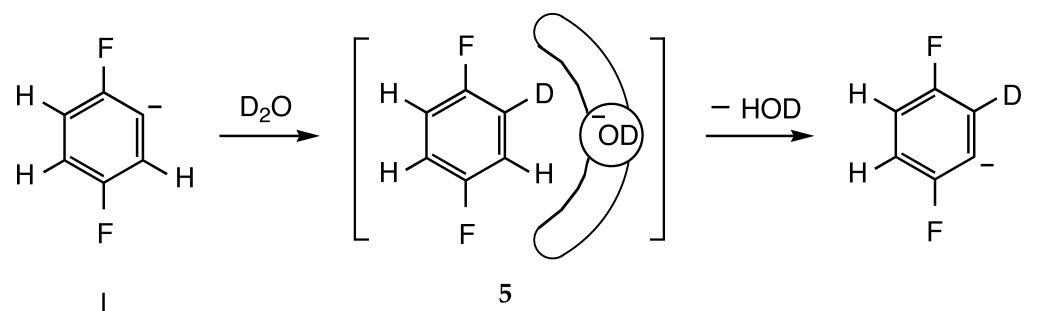<smiles>[2H]c1cc(F)c([13CH2]O)cc1F</smiles>

Scheme 3. Kinetically restricted $\mathrm{H} / \mathrm{D}$ exchange for the $p$-difluorophenyl anion with $\mathrm{D}_{2} \mathrm{O}$ and $\mathrm{CH}_{3} \mathrm{OD}$. 
the $\pi$ electrons on the top and bottom of the aromatic ring. For $\mathrm{H} / \mathrm{D}$ exchange with methanol the $\mathrm{CH}_{3} \mathrm{O}^{-}$ species can cross over to the other side of the ring by surmounting the $\pi$ electron barrier (6). The asterisks in 6 indicate the location of the methoxide anion at the "crossover" transition state. A similar transition state exists for hydroxide but the energy is higher than the reactants so the crossover does not take place. The number of exchangeable hydrogens thus provides information about the dynamics of ion-dipole intermediate complexes. On the other hand, these results caution against a simple interpretation of ion structure based solely on the extent of H/D exchange. ${ }^{78), 79)}$

\subsection{Termolecular association}

Mechanisms of gas phase ion-molecule termolecular association reactions can be probed by species- and site-specific deuteration of the reagents. For example, in the association reaction of methoxide and water molecules that is assisted by collisional stabilization with the helium buffer gas, how are the association rates affected by deuterium substitution of the bridging hydrogen (Eq. (18)) or the methyl group (Eq. (19)) relative to the perprotio system (Eq. (17))?

$$
\begin{aligned}
& \mathrm{CH}_{3} \mathrm{O}^{-}+\mathrm{H}_{2} \mathrm{O} \stackrel{\mathrm{He}}{\longrightarrow} \mathrm{CH}_{3} \mathrm{O}^{-} \cdot \mathrm{H}_{2} \mathrm{O} \\
& \mathrm{CH}_{3} \mathrm{O}^{-}+\mathrm{D}_{2} \mathrm{O} \stackrel{\mathrm{He}}{\longrightarrow} \mathrm{CH}_{3} \mathrm{O}^{-} \cdot \mathrm{D}_{2} \mathrm{O} \\
& \mathrm{CD}_{3} \mathrm{O}^{-}+\mathrm{H}_{2} \mathrm{O} \stackrel{\mathrm{He}}{\longrightarrow} \mathrm{CD}_{3} \mathrm{O}^{-} \cdot \mathrm{H}_{2} \mathrm{O}
\end{aligned}
$$

We systematically studied 14 association reactions of hydroxide-water, methoxide-water, methoxidemethanol, and ethoxide-ethanol and their deuterated analogs. ${ }^{80)}$ Apparent bimolecular rates are measured as a function of helium pressure to derive the rate constants for termolecular association. Association rates are significantly enhanced by deuteration of the alkyl groups (by factors of 1.4-3.0) whereas deuteration of the bridging hydrogen does not affect the association rates.

The observed isotope effects can be understood in terms of the structure and vibrational modes for the association complex. ${ }^{80}$ For example, in association of methoxide with water, the intermediate complex 7 formed from $\mathrm{CH}_{3} \mathrm{O}^{-}$and $\mathrm{H}_{2} \mathrm{O}$ (rate constant $k_{\mathrm{f}}$ ) is likely to have a hydrogen-bonded structure (Scheme 4). ${ }^{81), 82)}$ The energized complex either back-dissociates to the reactants (rate constant $k_{\mathrm{b}}$ ) or is collisionally stabilized to the adduct $\mathrm{CH}_{3} \mathrm{O}^{-} \cdot \mathrm{H}_{2} \mathrm{O}$ (rate constant $k_{\mathrm{s}}$, neglecting slow radiative stabilization). If the increase in the complex density of states overwhelms the increase in the reactant density of states upon deuterium substitution, the complex lifetime increases (i.e., $k_{\mathrm{b}}$ decreases) and more efficient adduct formation results. The observed lifetime increase upon deuteration of the methyl group is dominated by the increase of the density of states provided by the low frequency modes $(<200$ $\mathrm{cm}^{-1}$ ), which are due to methyl tortions and internal rotation. ${ }^{81)}$ On the other hand, deuterium substitution on the bridging position does not affect the complex lifetime because the high-frequency $\mathrm{O}-\mathrm{H}$ and $\mathrm{O}-\mathrm{D}$ vibrations ${ }^{83)}$ do not substantially increase the density of states. Detailed analysis of the magnitude and pressure dependence of the association rates has also provided insights into the potential energy surface (singleversus double-well) and dynamical bottleneck for ionmolecule association. ${ }^{80)}$

\section{Fundamental Ion Chemistry of Biological Interest}

\section{$5.1 \mathrm{~S}_{\mathrm{N}} 2$ reaction at nitrogen center}

Nucleophilic substitution at a nitrogen center is a convenient synthetic path to the formation of $\mathrm{C}-\mathrm{N}$ bonds. ${ }^{84}$ The reactions have also been recognized to play a significant role in carcinogenesis. ${ }^{85), 86)}$ The existence of a classical $\mathrm{S}_{\mathrm{N}} 2$ transition state at a nitrogen substrate has been inferred using double labeling experiments in the liquid phase, ${ }^{87)}$ and theoretically supported for a simple system $\mathrm{X}^{-}+\mathrm{H}_{2} \mathrm{NY} \rightarrow \mathrm{XNH}_{2}+$ $\left.\mathrm{Y}^{-} .88\right)$,89) However, no experimental data were available for the gas-phase kinetics of $\mathrm{S}_{\mathrm{N}} 2$ reactions at nitrogen centers.

We first investigated the $\mathrm{S}_{\mathrm{N}} 2$ reactions of chloramine $\left(\mathrm{NH}_{2} \mathrm{Cl}\right)$ with several anionic nucleophiles (Fig. 4c). ${ }^{90)}$ The unstable chloramine substrate was synthesized in situ and allowed to react with several nucleophiles in the flow tube $\left(\mathrm{Nu}^{-}=\mathrm{HO}^{-}, \mathrm{CH}_{3} \mathrm{O}^{-}, \mathrm{C}_{2} \mathrm{H}_{5} \mathrm{O}^{-}, \mathrm{C}_{3} \mathrm{H}_{7} \mathrm{O}^{-}, \mathrm{F}^{-}\right.$, $\mathrm{C}_{6} \mathrm{H}_{5} \mathrm{CH}_{2} \mathrm{O}^{-}, \mathrm{CF}_{3} \mathrm{CH}_{2} \mathrm{O}^{-}$, $\mathrm{HS}^{-}$, and $\mathrm{Cl}^{-}$, in the decreasing order of gas phase basicity). The most stable ion-molecule complex has the structure 8 , which directly correlates with the proton-transfer products $\mathrm{NuH}+$ $\mathrm{NHCl}^{-}$. In fact, proton transfer was observed for $\mathrm{C}_{3} \mathrm{H}_{7} \mathrm{O}^{-}$and more basic anions, leading to the assignment of the deprotonation enthalpy of chloramine ${ }^{90)}$ as $374.4 \pm 3.0 \mathrm{kcal} \mathrm{mol}^{-1}$. Nucleophilic substitution was observed for all reactant anions via the transition state 9 producing $\mathrm{NuNH}_{2}+\mathrm{Cl}^{-}$. The $\mathrm{S}_{\mathrm{N}} 2$ reactions are faster than the corresponding $\mathrm{S}_{\mathrm{N}} 2$ reactions of methyl chloride; the chloramine reactions take place at nearly every collision when the reaction is exothermic. Most dramatically, the symmetric $\mathrm{S}_{\mathrm{N}} 2$ reaction of ${ }^{37} \mathrm{Cl}^{-}+$ $\mathrm{H}_{2} \mathrm{~N}^{35} \mathrm{Cl}$ is found to be more than two orders of magnitude faster than the carbon-center analog of ${ }^{37} \mathrm{Cl}^{-}+$ $\mathrm{CH}_{3}{ }^{35} \mathrm{Cl}$ (Eq. (12)). There has been controversy on the $\mathrm{S}_{\mathrm{N}} 2$ barrier height $\Delta E^{\neq}$ovr at nitrogen relative to that at carbon. The significantly enhanced $\mathrm{S}_{\mathrm{N}} 2$ reactivity of

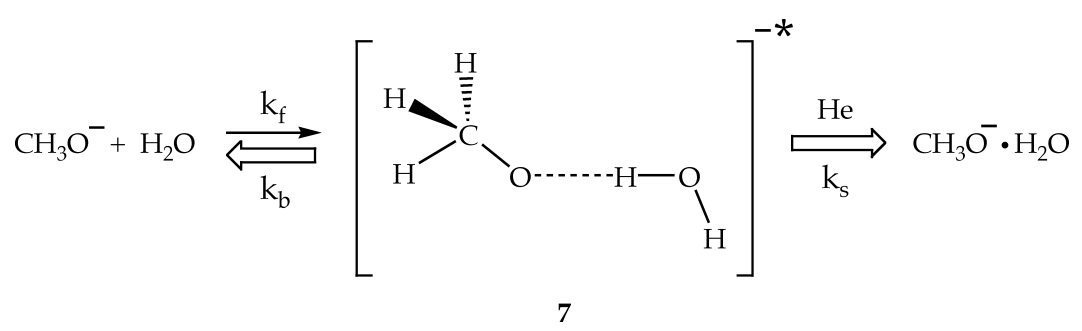

Scheme 4. Termolecular association of methoxide and water assisted by collision with He. 


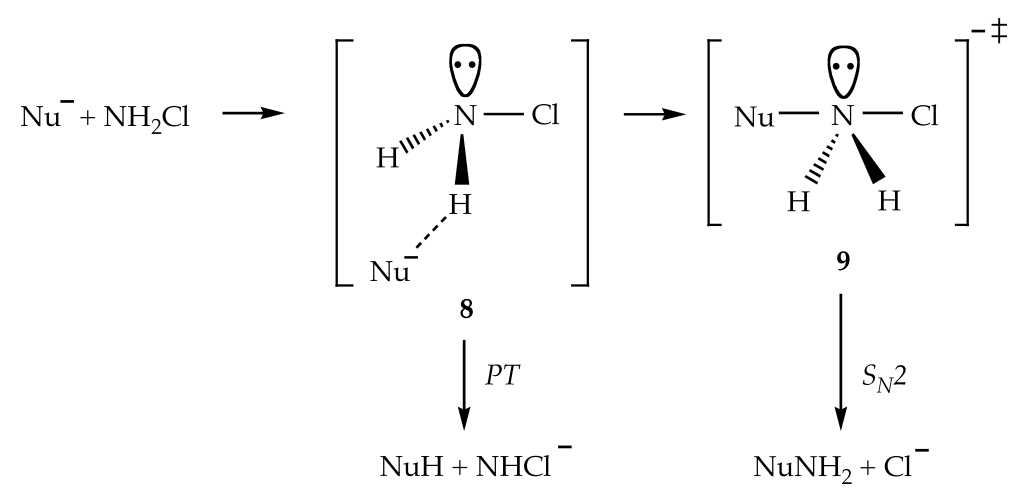

Scheme 5. Competitive proton transfer and $\mathrm{S}_{\mathrm{N}} 2$ reactions of chloramine with a nucleophile $\mathrm{Nu}^{-}$.

$\mathrm{NH}_{2} \mathrm{Cl}$ supports a high-level ab initio theoretical prediction $^{91)}$ that overall barrier heights for identity $\mathrm{S}_{\mathrm{N}} 2$ reactions $\mathrm{X}^{-}+\mathrm{NH}_{2} \mathrm{X}$ are negative and lower than for carbon-center $\mathrm{S}_{\mathrm{N}} 2$ reactions $\mathrm{X}^{-}+\mathrm{CH}_{3} \mathrm{X}$.

\section{$5.2 \quad \mathrm{E}_{\mathrm{CO}} 2$ elimination reactions}

For larger reactants such as propyl halides reacting with nucleophile anions, elimination at the $\beta$-carbon (E2) can compete with nucleophilic substitution at the $\alpha$-carbon (Eqs. (20) versus (21)).

$$
\mathrm{CH}_{3} \mathrm{CH}_{2} \mathrm{CH}_{2} \mathrm{X}+\mathrm{Nu}^{-} \stackrel{\mathrm{S} 2}{\stackrel{\mathrm{S} 2}{\longrightarrow} \mathrm{CH}_{3} \mathrm{CH}_{2} \mathrm{CH}_{2} \mathrm{Nu}+\mathrm{X}_{3}^{-} \mathrm{CH}=\mathrm{CH}_{2}+\mathrm{NuH}+\mathrm{X}^{-}}
$$

These two pathways yield the same halide anion $\mathrm{X}^{-}$ and hence cannot be distinguished directly by product ion analysis. Relative importance of $\mathrm{S}_{\mathrm{N}} 2$ versus $\mathrm{E} 2$ channels was deduced from systematic measurements of rate constants for $\alpha$-branched alkyl halides. ${ }^{92)}$ It was concluded that the $\mathrm{S}_{\mathrm{N}} 2$ channel dominates with sulfur anions, whereas the reaction proceeds mainly via the E2 channel with oxo anions. ${ }^{92)}$ Deuterium KIEs have also been measured using the SIFT technique to differentiate the two mechanisms. ${ }^{93)}$ More recently an ingenious method has been devised that uses dianions as attacking nuculeophiles, ${ }^{94)}$ thereby leaving a negative charge on the (otherwise) neutral product and allowing for its mass spectrometric detection.

We recently used the SIFT technique to study a different type of elimination reactions (" $\mathrm{E}_{\mathrm{CO}} 2$ "), in which alkyl hydroperoxides react with gas phase anions to form $\mathrm{C}=\mathrm{O}$ double bonds in the product. ${ }^{95)}$ In the rapid reaction of methyl hydroperoxide with fluoride $(49 \%$ efficiency with respect to the collision rate, Scheme 6), formation of hydroxide via complex 11 is the major pathway $(\approx 85 \%)$ whereas slightly endothermic proton transfer via complex $\mathbf{1 0}$ is minor $(\approx$ 10\%). Nucleophilic substitution at the methyl group to form $\mathrm{HOO}^{-}$is endothermic and is not observed. The $\mathrm{E}_{\mathrm{CO}} 2$ reaction proceeds via anionic attack on an $\alpha$-hydrogen followed by concerted elimination of formaldehyde and $\mathrm{HO}^{-}$. The mechanism is substantiated by observing $\mathrm{HO}^{-}$as the major product from deuterated reactant $\mathrm{CD}_{3} \mathrm{OOH}$. The importance of the $\alpha$-hydrogen is demonstrated by the formation of hydroxide from $\mathrm{CH}_{3} \mathrm{OOH}$ and $\mathrm{CH}_{3} \mathrm{CH}_{2} \mathrm{OOH}$ and not from $\left.\left(\mathrm{CH}_{3}\right)_{3} \mathrm{COOH} .{ }^{95}\right)$

Energy and structure calculations support the mechanism and high efficiency for the $\mathrm{E}_{\mathrm{CO}} 2$ reaction (Fig. 7).

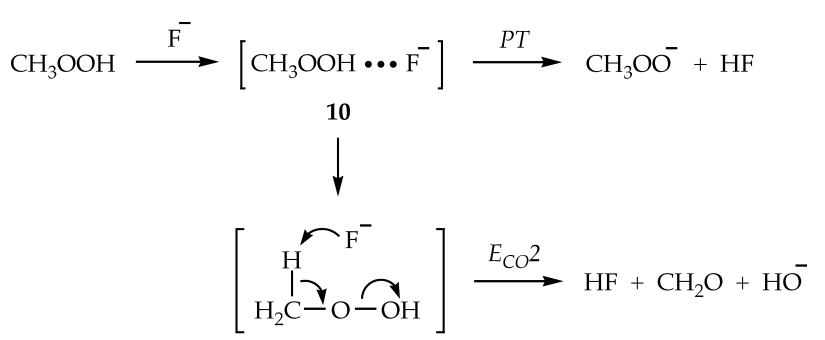

11

Scheme 6. Competitive proton transfer and $\mathrm{E}_{\mathrm{CO}} 2$ reactions of methyl hydroperoxide with fluoride.

The $\mathrm{CH}_{3} \mathrm{OOH}+\mathrm{F}^{-}$reaction proceeds via initial formation of complex 11 with the fluoride associated with one of the $\alpha$-hydrogens, then surmounting the key transition state TS1 which is nearly $15 \mathrm{kcal} \mathrm{mol}^{-1}$ lower in energy than the reactants. Consistent with the concerted nature of the reaction, the $\mathrm{E}_{\mathrm{CO}} 2$ transition state is an antiperiplanar structure with $\mathrm{C}-\mathrm{H}$ and O-O bonds elongated by about $0.2 \AA$ relative to free $\mathrm{CH}_{3} \mathrm{OOH}$ while the $\mathrm{C}-\mathrm{O}$ bond is contracting. ${ }^{95)}$ The reverse reaction, $\mathrm{CH}_{3} \mathrm{OO}^{-}+\mathrm{HF}$, is found to proceed at nearly every collision and yields $\mathrm{HO}^{-}$as the major primary reaction product $(\approx 80 \%)$, despite the possibility for exothermic proton transfer. This is also totally consistent with the computation that the reactants can follow the same pathway as above after the initial complex $\mathbf{1 0}$ has surmounted the transition state TS2 (Fig. 7).

The gas phase ion study has unambiguously revealed the mechanism and importance of base-mediated decomposition of alkyl hydroperoxides, which has been controversial in solution. ${ }^{96), 97)}$ These results also bear biological implications associated with degradation of lipid hydroperoxides producing genotoxic aldehydes and ketones. ${ }^{98)}$,99) In contrast to fluoride, a weaker base such as $\mathrm{NC}^{-}$cannot induce the $\mathrm{E}_{\mathrm{CO}} 2$ reaction with $\mathrm{CH}_{3} \mathrm{OOH} .{ }^{95}$ ) However, our preliminary calculations suggest that as the acidity of the $\alpha$-hydrogen increases, weak bases such as cyanide and chloride may facilitate $\mathrm{E}_{\mathrm{CO}} 2$ decomposition. Those peroxides include allyl hydroperoxide $\left(\mathrm{CH}_{2}=\mathrm{CHCH}_{2} \mathrm{OOH}\right)$ and conjugated diene hydroperoxides, good models for endogenous biological lipid hydroperoxides. 


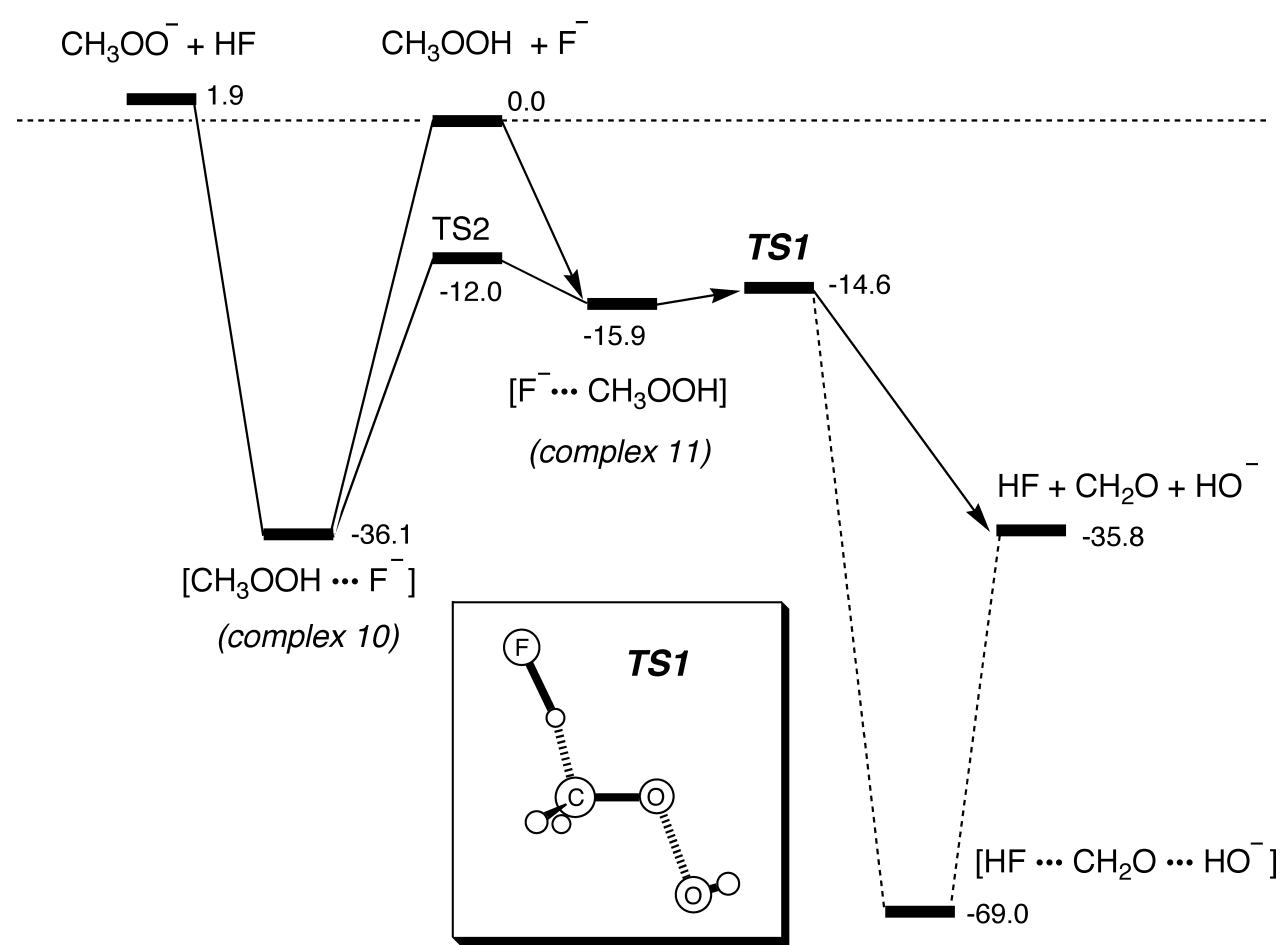

Fig. 7. Relative energies $\left(\mathrm{kcal} \mathrm{mol}^{-1}\right)$ on the $\left[\mathrm{F}^{-}, \mathrm{CH}_{3} \mathrm{OOH}\right]$ potential energy surface (electronic $+\mathrm{ZPE}$ ). Data taken from Blanksby et al. ${ }^{95)}$ The inset shows the structure of the $\mathrm{E}_{\mathrm{CO}} 2$ transition state, TS1.

We also studied collision-induced dissociation of the $[\mathrm{M}-\mathrm{H}]^{-}$anions of alkyl hydroperoxides, $\mathrm{CH}_{3} \mathrm{OO}^{-}$, $\mathrm{CH}_{3} \mathrm{CH}_{2} \mathrm{OO}^{-}$, and $\left(\mathrm{CH}_{3}\right)_{3} \mathrm{COO}^{-}{ }^{100)}$ Facile decomposition of ethyl- and $t$-butyl peroxide anions to produce $\mathrm{HOO}^{-}$ was observed. Dehydration of peroxide anions was also observed but it is a minor process occurring only at high collision energies. ${ }^{100)}$ This is in contrast to the CID of the $[\mathrm{M}-\mathrm{H}]^{-}$anions of lipid hydroperoxides, in which loss of water is the key initial step in the decomposition. ${ }^{101)}$ Combining the results of deuterium labeling, ${ }^{101)}$ we propose that decomposition of lipid peroxide anions actually proceeds via an intramolecular $\mathrm{E}_{\mathrm{CO}} 2$ mechanism. ${ }^{100)}$ Incorporation of a powerful electrospray ionization (ESI) source in the flow tube ${ }^{7)}$ is expected to allow studies of large peroxides with the SIFT setup. We are currently developing those ESI sources.

\section{Structure and Thermochemistry of Energetic and Unstable Anions}

\subsection{Peroxide anions}

Hydroperoxyl (HOO) and alkyl peroxyl (ROO) radicals are key intermediates in combustion. HOO radicals also play a vital role in oxidation of volatile organic compounds in atmosphere. ROO radicals are usually the first oxidation products of hydrocarbons in troposphere, resulting in ozone formation through photochemical cycles. Heats of formation of the peroxyl radicals are crucial in modeling these processes, and yet the experimental values have been controversial and largely unestablished (these aspects reviewed and discussed in Blanksby et al. ${ }^{102)}$ and Ramond et al. ${ }^{103)}$ ).

To accurately determine the peroxyl radical heats of formation, we used the acidity/electron affinity thermochemical cycle ${ }^{104)}$ which combines the gas phase acidity measurements with SIFT and electron affinity measurements with negative ion photoelectron spec- troscopy (NIPES). ${ }^{102), 103)}$ The gas phase acidity of alkyl hydroperoxide, $\Delta_{\text {acid }} G_{298}(\mathrm{ROOH})$, is determined at room temperature by measuring the forward $\left(k_{\mathrm{f}}\right)$ and reverse $\left(k_{\mathrm{r}}\right)$ rate constants for the proton-transfer equilibrium between the peroxide anion and a reference acid (HA) (Eq. (22)).

$$
\mathrm{ROO}^{-}+\mathrm{HA} \underset{k_{\mathrm{r}}}{\stackrel{k_{\mathrm{f}}}{\gtrless}} \mathrm{ROOH}+\mathrm{A}^{-}
$$

The equilibrium constant $K_{\text {equil }}\left(=k_{\mathrm{f}} / k_{\mathrm{r}}\right)$ is connected to $\Delta_{\text {acid }} G_{298}(\mathrm{ROOH})$ through a known acidity value for HA (Eq. (23)). The enthalpy of deprotonation, $\Delta_{\text {acid }} H_{298}$ $(\mathrm{ROOH})$, is then derived using computational entropies.

$$
\begin{aligned}
\Delta_{\text {rxn }} G_{298} & =\Delta_{\text {acid }} G_{298}(\mathrm{HA})-\Delta_{\text {acid }} G_{298}(\mathrm{ROO}-\mathrm{H}) \\
& =-R T \ln K_{\text {equil }}
\end{aligned}
$$

The electron affinity of the peroxyl radical, $E A(\mathrm{ROO})$, is measured by NIPES of the $\mathrm{ROO}^{-}$anion. The measured deprotonation enthalpy and electron affinity, along with the ionization energy of hydrogen $I E(\mathrm{H})$, afford the bond dissociation enthalpy, $\mathrm{DH}_{298}(\mathrm{ROO}-\mathrm{H})$ (Eq. (24)). The thermal correction term arises from the fact that $E A(\mathrm{ROO})$ and $I E(\mathrm{H})$ are measured at $0 \mathrm{~K}$. The correction is usually very small $\left(<0.3 \mathrm{kcal} \mathrm{mol}^{-1}\right)$ and can be neglected, ${ }^{104)}$ or computed from the relevant heat capacities. The $0 \mathrm{~K}$ bond dissociation energy, $D H_{0}$ (ROO-H), is also calculated from the $298 \mathrm{~K}$ bond enthalpy following similar thermal corrections.

$$
\begin{array}{r}
D H_{298}(\mathrm{ROO}-\mathrm{H})=\Delta_{\text {acid }} H_{298}(\mathrm{ROO}-\mathrm{H})-I E(\mathrm{H}) \\
+E A(\mathrm{ROO} \bullet)+(\text { thermal correction })
\end{array}
$$

Finally, the heat of formation for ROO is obtained from the bond enthalpy and known heats of formation for hydrogen and ROOH (Eq. (25)), without the need for relying on thermochemistry of other intermediate species. 
Table 1. Experimental Deprotonation Enthalpies, Electron Affinities, Bond Dissociation Energies, and Heats of Formation for Alkyl Peroxyl Radicals, ROO, and Hydroperoxides, $\mathrm{ROOH}^{\mathrm{a}}$

\begin{tabular}{lccrr}
\hline \multicolumn{1}{c}{$\mathrm{R}$} & $\begin{array}{c}\Delta_{\mathrm{acid}} H_{298}(\mathrm{ROOH}) \\
\left(\mathrm{kcal} \mathrm{mol}{ }^{-1}\right)\end{array}$ & $\begin{array}{c}E A(\mathrm{ROO}) \\
(\mathrm{eV})\end{array}$ & $\begin{array}{c}D H_{0}(\mathrm{ROO}-\mathrm{H}) \\
\left(\mathrm{kcal} \mathrm{mol}^{-1}\right)\end{array}$ & $\begin{array}{r}\Delta_{\mathrm{f}} H_{298}(\mathrm{ROO}) \\
\left(\mathrm{kcal} \mathrm{mol}^{-1}\right)\end{array}$ \\
\hline $\mathrm{H}$ & $376.5 \pm 0.4$ & $1.078 \pm 0.006$ & $86.6 \pm 0.5$ & $3.2 \pm 0.5$ \\
$\mathrm{CH}_{3}$ & $374.6 \pm 1.0$ & $1.161 \pm 0.005$ & $86.9 \pm 1.0$ & $4.8 \pm 1.2$ \\
$\mathrm{CH}_{3} \mathrm{CH}_{2}$ & $371.0 \pm 2.2$ & $1.186 \pm 0.004$ & $83.9 \pm 2.2$ & $-6.8 \pm 2.3$ \\
$\left(\mathrm{CH}_{3}\right)_{3} \mathrm{C}$ & $370.2 \pm 2.1$ & $1.196 \pm 0.011$ & $83.3 \pm 2.1$ & $-25.2 \pm 2.5$ \\
\hline
\end{tabular}

a) Ramond et al. ${ }^{103)}$ for $\mathrm{R}=\mathrm{H}$ and Blanksby et al. ${ }^{102)}$ for $\mathrm{R}=\mathrm{CH}_{3}, \mathrm{CH}_{3} \mathrm{CH}_{2}$, and $\left(\mathrm{CH}_{3}\right)_{3} \mathrm{C}$.

$$
\begin{aligned}
\Delta_{\mathrm{f}} H_{298}(\mathrm{ROO} \bullet & =D H_{298}(\mathrm{ROO}-\mathrm{H})-\Delta_{\mathrm{f}} H_{298}(\mathrm{H}) \\
& +\Delta_{\mathrm{f}} H_{298}(\mathrm{ROOH})
\end{aligned}
$$

Table 1 summarizes the thermochemical parameters for $\mathrm{HOO}, \mathrm{CH}_{3} \mathrm{OO}, \mathrm{CH}_{3} \mathrm{CH}_{2} \mathrm{OO}$, and $\left(\mathrm{CH}_{3}\right)_{3} \mathrm{COO}$ radicals determined to within an accuracy of a few $\mathrm{kcal} \mathrm{mol}^{-1}$ or better. The uncertainties in bond dissociation energies and heats of formation arise primarily from those in the measured heats of deprotonation. It is noted that the SIFT measurement of the relative acidity $\left(\Delta_{\mathrm{rxn}} G_{298}\right)$ is very accurate to within a fraction of kcal $\mathrm{mol}^{-1}$, and the uncertainty in $\Delta_{\text {acid }} H_{298}(\mathrm{ROOH})$ is almost entirely due to the error bars in the reported gas phase acidities for the reference acids (Eq. (23)). The heat of formation for $\mathrm{HOO}$ is nearly $3 \mathrm{kcal} \mathrm{mol}^{-1}$ higher ${ }^{103)}$ than the previous recommended value ${ }^{105)}$ of $0.5 \pm 2.0 \mathrm{kcal} \mathrm{mol}{ }^{-1}$, but is consistent with several recent values obtained using different techniques (reviewed in Ramond et al. ${ }^{103)}$ ). The measured heats of formation for the alkyl peroxyl radicals ${ }^{102)}$ also agree with several previous reports, settling the controversy in the thermochemistry of these reactive species.

\subsection{Nitrogen-containing anions}

Compounds containing many nitrogen atoms or nitrogen substituents (e.g., $-\mathrm{NO}_{2}$ and $-\mathrm{N}_{3}$ groups) are energetic species. Anions and radicals of those species are also considered important intermediates in combustion. The SIFT technique has been employed to study, through an anionic approach, the structure and thermochemistry of diazocarbene $(\mathrm{CNN})$ and the diazomethyl radical $(\mathrm{HCNN}),{ }^{106)}$ small nitroalkanes, ${ }^{107)}$ and a series of five-membered azole compounds. ${ }^{108)}$

$\mathrm{HCNN}^{-}$and $\mathrm{CNN}^{--}$anions can be generated, for example, by $\mathrm{HO}^{-}$deprotonation and $\mathrm{O}^{--}$dehydrogena- tion of diazomethane, respectively (Scheme 7). Proton affinities of these anions were determined by protontransfer equilibrium (for $\mathrm{HCNN}^{-}$) and acidity bracketing (for $\mathrm{CNN}^{--}$) measurements, to be $372 \pm 2$ and 352 $\pm 4 \mathrm{kcal} \mathrm{mol}^{-1}$, respectively. ${ }^{106)}$ It is noted that only acidity bracketing is feasible with $\mathrm{CNN}^{--}$because the reverse reaction requires the $\mathrm{HCNN}$ radical reactant. Photodetachment of $\mathrm{HCNN}^{-}$and $\mathrm{CNN}^{-}$gives access to $[\mathrm{HCNN}]$ and $[\mathrm{CNN}]$, which are conjectured to be combustion intermediates that provide low energy paths to cleave $\mathrm{N}_{2}$ to produce $\mathrm{N}$ atoms (Scheme 7). The nitrogen atoms are rapidly oxidized to nitric oxide, a major source driving urban air pollution. Electron affinities of HCNN and CNN were measured by NIPES of these anions. Given the heat of formation of diazomethane, heats of formation for the HCNN radical and CNN carbene were accurately determined. It turns out that the HCNN intermediate provides a deep well of $-31 \pm 3 \mathrm{kcal} \mathrm{mol}^{-1}$ relative to the $\mathrm{CH}+\mathrm{N}_{2}$ reactants in

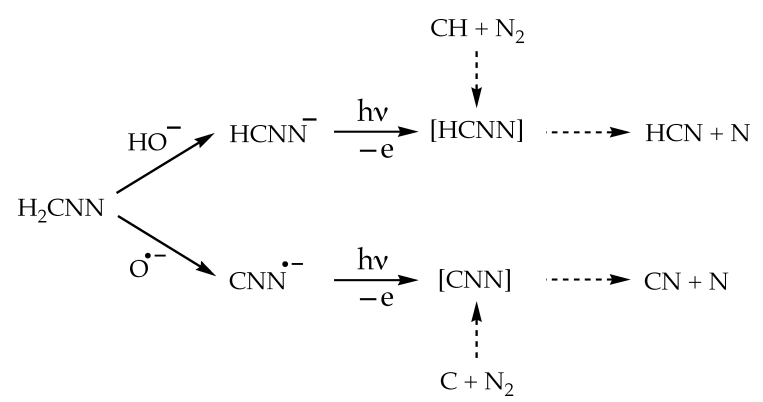

Scheme 7. Formation of HCNN and CNN intermediates using gas phase ion schemes (solid arrows), along with formation and subsequent dissociation in combustion (broken arrows).

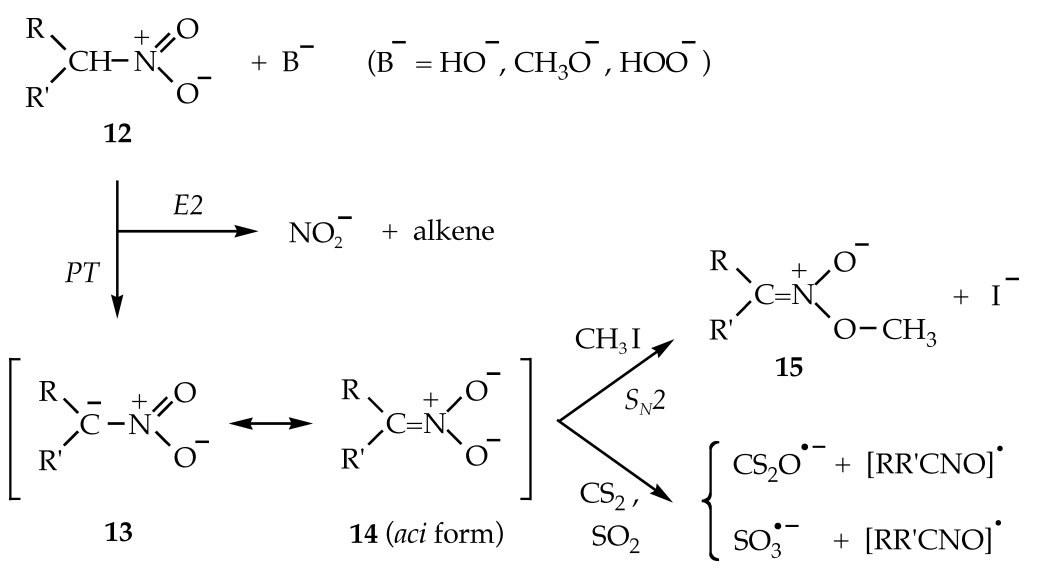

Scheme 8. Competitive proton transfer and E2 elimination reactions of nitroalkane, and the chemical reactivity of the deprotonated nitroalkane anion. 
the spin-forbidden $\mathrm{CH}\left({ }^{2} \mathrm{\Pi}\right)+\mathrm{N}_{2} \rightarrow \mathrm{HCN}+\mathrm{N}\left({ }^{4} \mathrm{~S}\right)$ reaction, which is slightly endothermic, $\Delta_{\mathrm{rxn}} H_{0}=1.6 \pm 0.7$ kcal $\mathrm{mol}^{-1}$. The detailed mechanism of how the doublet/quartet crossing occurs within the deep potential well remains an intriguing question.

Nitro compounds are prototypical propellants and explosives, as exemplified by high-performance explosives trinitrotoluene (TNT) and 1,3,5-trinitro-1,3,5triazacyclohexane (RDX). Apart from acidity determinations, reactivity studies of nitroalkanes and their anions are relatively few in the gas phase. We are especially interested in the stability and reactivity of deprotonated nitroalkanes (aci-anions), which have been considered a key intermediate in the condensed phase, base-sensitized detonation of nitroalkanes. 109)-111) Small nitroalkanes (nitromethane, nitroethane, 2-nitropropane, 2-methyl-2-nitropropane, and nitrocyclopropane) were allowed to react with gas phase bases $\mathrm{HO}^{-}, \mathrm{CH}_{3} \mathrm{O}^{-}$, and $\mathrm{HOO}^{-}$. We find the reactions to proceed very rapidly, $k=2.5-4.3 \times 10^{-9} \mathrm{~cm}^{3} \mathrm{~s}^{-1}$, primarily producing $[\mathrm{M}-\mathrm{H}]^{-}$anions via proton transfer (where an $\alpha$-hydrogen is available) as well as $\mathrm{NO}_{2}{ }^{-}$via the E2 pathway (where a $\beta$-hydrogen is available). ${ }^{107)}$ $\mathrm{S}_{\mathrm{N}} 2$ formation of $\mathrm{NO}_{2}{ }^{-}$is ruled out because $\mathrm{NO}_{2}{ }^{-}$is not observed from $\mathrm{CH}_{3} \mathrm{NO}_{2}$. The acyclic nitroalkanes are similarly very acidic because of resonance stabilization involving the aci form $\mathbf{1 4}$ for the deprotonated anions, in addition to the strong inductive effect by the nitro group. ${ }^{12)}$ The anomalously low acidity for nitrocyclopropane, which has the $\mathrm{R}-\mathrm{R}^{\prime}$ bonding, is explained by the destabilizing strain present in the aci form.

Reactivity studies revealed the importance of the aci form in the $[\mathrm{M}-\mathrm{H}]^{-}$anions of acyclic nitroalkanes. ${ }^{107)}$ In the $\mathrm{S}_{\mathrm{N}} 2$ reactions of $[\mathrm{M}-\mathrm{H}]^{-}$anions with $\mathrm{CH}_{3} \mathrm{I}$, the efficiencies do not decrease but rather gradually increase with larger substituents $\mathrm{R}$ and $\mathrm{R}^{\prime}$ (Scheme 8).
This is an interesting result because the larger attacking anions have greater crowdedness ${ }^{67)}$ around the deprotonated carbon atom and hence are expected to display a reduced $\mathrm{S}_{\mathrm{N}} 2$ reactivity. The observation is rationalized if the nucleophilic attack is actually made by the oxo anion in the aci form (Scheme 8). The major products $\mathrm{CS}_{2} \mathrm{O}^{--}$and $\mathrm{SO}_{2}{ }^{--}$observed from the reactions with $\mathrm{CS}_{2}$ and $\mathrm{SO}_{2}$, respectively, are also consistent with the aci form being the important resonant structure. The $[\mathrm{M}-\mathrm{H}]^{-}$anion of nitrocyclopropane displays different $\mathrm{S}_{\mathrm{N}} 2$ and bimolecular reactivities than do acyclic anions, which is explained by the reduced contribution of the aci form in the cyclic anion. ${ }^{107)}$

Polynitrogen compounds have been of both fundamental and practical interests. ${ }^{113)}$ In theory those species can derive from isoelectronic substitution of $\mathrm{N}$ atoms for $\mathrm{CH}$ units; however, polynitrogen compounds are considerably less stable because of the nitrogen lone pair repulsion that is introduced. Only a handful of homonuclear polynitrogen species has been discovered, ${ }^{113)}$ e.g., azide $\left(\mathrm{N}_{3}{ }^{-}\right)$. This, in turn, indicates that these species are promising high energy density materials (HEDM). For example, five-membered rings pentazole $\left(\mathrm{HN}_{5}\right)$ and the pentazolide anion $\left(\mathrm{N}_{5}{ }^{-}\right)$have been predicted to be metastable. ${ }^{114), 115)}$ In fact, the $\mathrm{N}_{5}{ }^{-}$anion $\left(\Delta H_{\mathrm{f}}=62 \pm 4 \mathrm{kcal} \mathrm{mol}^{-1}\right)^{114)}$ has recently been generated in the gas phase by CID $^{116)}$ and laser ablation ${ }^{117}$ of appropriate precursor molecules containing $\mathrm{N}_{5}$ units. $\mathrm{N}_{5}{ }^{-}$has also been formed as a zinc salt in a $-40^{\circ} \mathrm{C}$ solution. ${ }^{118)}$

Despite the observation of $\mathrm{N}_{5}{ }^{-}$anions, experimental characterization of $\mathrm{N}_{5}{ }^{-}$and its derivatives has been extremely difficult. We have taken a different approach in which we study deprotonated anions of lower azoles in the gas phase by measuring the proton affinity $(P A)$ and electron binding energy $(E B E)$, then

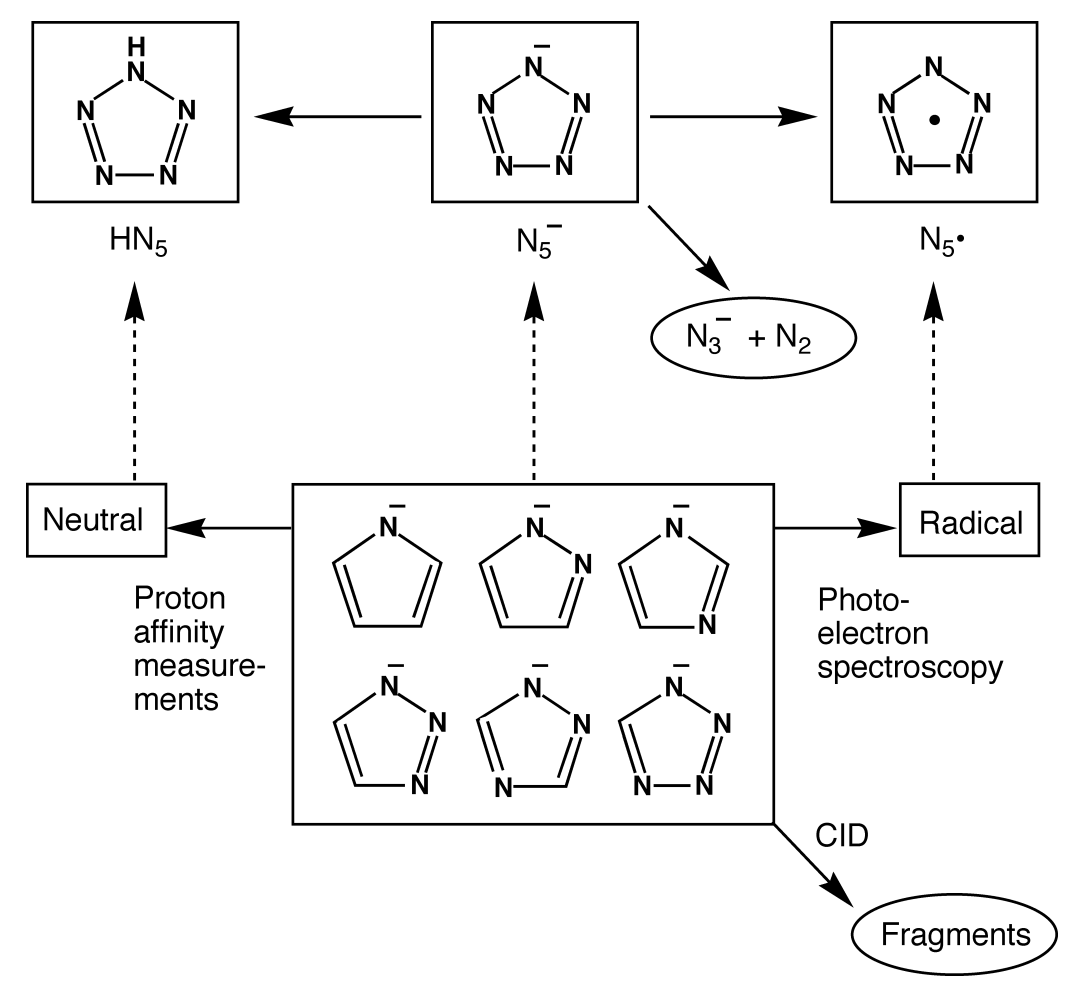

Fig. 8. Pentazole $\left(\mathrm{HN}_{5}\right)$, pentazolide anion $\left(\mathrm{N}_{5}^{-}\right)$, pentazolyl radical $\left(\mathrm{N}_{5} \bullet\right.$ ), and lower azolide anions $\left(\mathrm{N}_{1}-\mathrm{N}_{4}\right)$. 
extrapolate the results to the pentazole family (Fig. 8). CID threshold energies and fragmentation patterns can also be examined for these lower azolide anions in order to evaluate the pentazolide stability against metastable decomposition: $\mathrm{N}_{5}{ }^{-} \rightarrow \mathrm{N}_{3}{ }^{-}+\mathrm{N}_{2}$. We have generated all azolide anions in Fig. 8. While this is a relatively new project with most of the work currently in progress, our first experiments on the pyrrolide anion $\left(\mathrm{C}_{4} \mathrm{H}_{4} \mathrm{~N}^{-}\right)^{108)}$ have determined its proton affinity and electron binding energy. The anion is very stable $(E B E=2.145 \pm 0.010 \mathrm{eV})$, and strong vibronic coupling is indicated between the ground and first excited states for the detached pyrrolyl radical. ${ }^{108)}$ The SIFT instrument is also being used for CID studies of these azolide anions. Preliminary experiments indicate an increasing trend for $E B E$ and decreasing trends for $\Delta_{\text {acid }} H_{298}$ and the CID threshold as the heterocyclic ring incorporates more nitrogen atoms.

\subsection{Cyclooctatetraene and cyclopentanone}

Conformational changes in 1,3,5,7-cyclooctatetraene $\left(\mathrm{COT}, \mathrm{C}_{8} \mathrm{H}_{8}\right)$ and its substituted derivatives have been a subject of considerable research interest for several decades; tub-like COT $\left(D_{2 \mathrm{~d}}\right)$ undergoes ring inversion via a planar $D_{8 \mathrm{~h}}$ structure. ${ }^{119), 120)}$ Relatively little is known, however, about the thermochemical properties of COT and, in particular, its radical and anionic derivatives. We generated anionic derivatives of COT using electron impact, $\mathrm{HO}^{-}$deprotonation, and the $\mathrm{O}^{-}$reaction to abstract $\mathrm{H}_{2}{ }^{+}$(Scheme 9). Determination of the adiabatic electron affinity $(E A)$ of COT has been challenging for many years because of the substantial conformational change $\left(D_{2 \mathrm{~d}} \rightarrow D_{4 \mathrm{~h}}\right)$ upon electron attachment $\left[\mathrm{COT}+\mathrm{e} \rightarrow \mathrm{COT}^{-}(\mathbf{1})\right]$. In fact, photodetachment of $\mathrm{COT}^{--}$has been shown to leave COT in a planar transition state for the ring inversion, ${ }^{121)}$ with no Franck-Condon overlap with the neutral vibrational ground state. We measured both the electron-transfer equilibrium and forward/reverse kinetic rates for the $\mathrm{COT}+\mathrm{O}_{2}{ }^{--} \rightleftarrows \mathrm{COT}^{--}+\mathrm{O}_{2}$ system. Under a truly thermal condition, the adiabatic EA of COT was determined unambiguously as $0.55 \pm 0.02 \mathrm{eV},{ }^{122)}$ in excel- lent agreement with later measurements using the kinetic method ${ }^{123)}$ and thermal-energy electron attachment/detachment, ${ }^{124)}$ and with high-level theoretical calculations. ${ }^{125)}$

The radical anion of cyclooctatetraene $\left[\operatorname{COT}^{-}(\mathbf{1})\right]$ is one of the first open-shell annulenes observed experimentally. The gas phase proton affinity for $\mathrm{COT}^{--}(\mathbf{1})$, along with those for $\mathrm{C}_{8} \mathrm{H}_{7}{ }^{-}(2 \boldsymbol{\alpha})$ and $\mathrm{C}_{8} \mathrm{H}_{6}{ }^{-}{ }^{-}(3)$, was also measured. Combining the values of proton affinities with electron binding energies measured using photoelectron spectroscopy, we determined the $\mathrm{C}-\mathrm{H}$ bond dissociation energies of COT and its derivatives, $\mathrm{C}_{8} \mathrm{H}_{n}$ $(n=7-10) .{ }^{122), 126)}$ Photoelectron spectroscopy and molecular orbital calculations revealed that the $\mathrm{C}_{8} \mathrm{H}_{7}{ }^{-}(2 \alpha)$ anion is a novel structure incorporating a $\pi$-electronic system and a bent allenic moiety within the eightmembered ring. ${ }^{122)}$

The large $\mathrm{C}_{8}$ ring systems undergo unexpected transformations upon collisional excitation. When SIFTinjected at high collision energies, $\mathrm{COT}^{-}{ }^{-}(\mathbf{1})$ and $\mathrm{C}_{8} \mathrm{H}_{6}{ }^{\cdot-}$ (3) radical anions appear to detach an electron for signal loss. Remarkably, the $\mathrm{C}_{8} \mathrm{H}_{7}{ }^{-}(2 \alpha)$ anion undergoes a collision-induced isomerization to $\mathrm{C}_{8} \mathrm{H}_{7}{ }^{-}(2 \beta)$ via transannular bridging (Scheme 9), ${ }^{126)}$ instead of usual ring opening or fragmentation. Measured chemical reactivity and molecular orbital calculations characterized $\mathrm{C}_{8} \mathrm{H}_{7}{ }^{-}(2 \boldsymbol{\beta})$ as a fairly stable bicyclic species $\left(\Delta H_{\mathrm{f}}=\right.$ $33 \mathrm{kcal} \mathrm{mol}^{-1}$ ) with $E B E$ and $P A$ very similar to those for cyclopentadienide anion. Duplication of this transannular bond formation process in solution would provide entry into bicyclic ring systems that pose significant synthetic challenge.

At even higher SIFT injection energies the $\mathrm{C}_{8} \mathrm{H}_{7}{ }^{-}(\boldsymbol{\beta} \boldsymbol{\beta})$ product is replaced by a $\mathrm{C}_{8} \mathrm{H}_{6}{ }^{\cdot-}(\boldsymbol{\gamma})$ species, apparently following loss of a hydrogen atom. This species exhibits distinctly different chemical reactivities from the isomeric $\mathrm{C}_{8} \mathrm{H}_{6} \cdot{ }^{-}(3)$. If it retains the skeletal structure of $\mathrm{C}_{8} \mathrm{H}_{7}{ }^{-}(2 \boldsymbol{\beta})$, this species may be the radical anion of pentalene, a prototypical Hückel antiaromatic molecule which eluded chemists for several decades. ${ }^{127)}$ With appropriate tuning of the injection energy, the

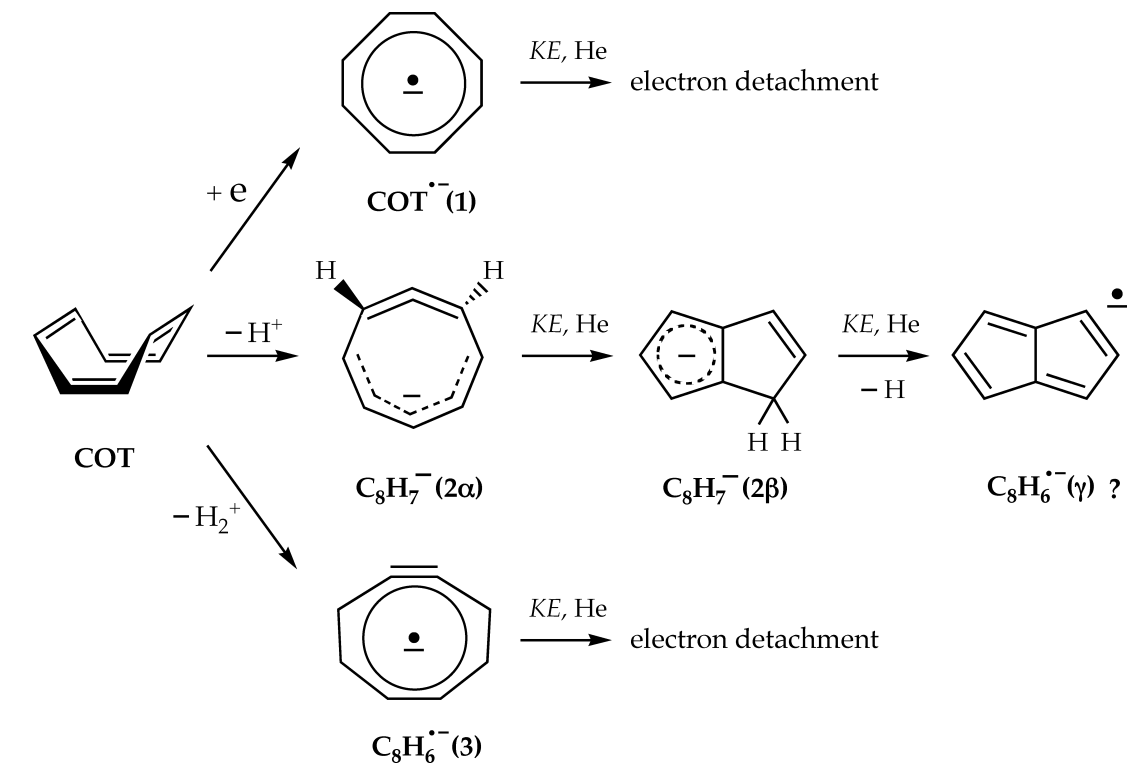

Scheme 9. Cyclooctatetraene, its anionic derivatives, and their collision-induced transformations. 


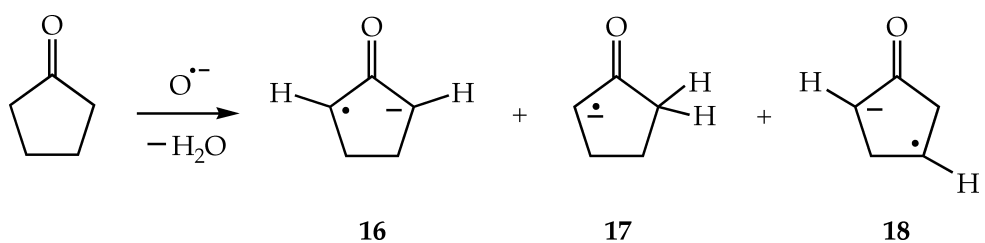

Scheme 10. Anions formed from the reaction of cyclopentanone with the oxygen radical anion.

$\mathrm{C}_{8} \mathrm{H}_{7}{ }^{-}(2 \boldsymbol{\alpha})$ anion is completely converted to $\mathrm{C}_{8} \mathrm{H}_{7}{ }^{-}(2 \boldsymbol{\beta})$ with minimal fragmentation to $\mathrm{C}_{8} \mathrm{H}_{6}{ }^{-}{ }^{-}(\boldsymbol{\gamma})$. The $\mathrm{C}_{8} \mathrm{H}_{7}{ }^{-}$ $(2 \boldsymbol{\beta})$ anion can then be subjected to CID under a single collision condition with the triple quadrupole (Fig. 1). Interestingly, formation of $\mathrm{C}_{8} \mathrm{H}_{6}{ }^{\cdot}(\boldsymbol{\gamma})$ has been very difficult to observe under experimental conditions where similar $m / z$ ions are easily being detected. This result may suggest that $\mathrm{C}_{8} \mathrm{H}_{6}{ }^{-}{ }^{-}(\boldsymbol{\gamma})$ can only be stabilized in the high-pressure collisional environment in the flow tube. Pressure-dependent product branching has been observed by a separate group. ${ }^{128)}$

As illustrated above, the atomic oxygen radical anion $\left(\mathrm{O}^{--}\right)$reacts with many organic compounds, formally abstracting $\mathrm{H}_{2}{ }^{+}$and producing a variety of carbene and distonic radical anions ${ }^{129), 130)}$ as well as anions with bent triple bonds. ${ }^{131)}$ We have shown that $\mathrm{O}^{--}$reacts with cyclopentanone to form a mixture of three $\mathrm{C}_{5} \mathrm{H}_{6} \mathrm{O}^{-}$isomers (Scheme 10). ${ }^{132)}$ The major product cyclopentanone-2,5-diyl radical anion (16) is thermodynamically most stable. Photodetachment of this species will determine the singlet-triplet splitting of the 2,5-diyl radical, an ethano-bridged analog of oxyallyl which has been postulated as an intermediate in many organic reactions. NIPES experiments are under way in the laboratory of Prof. W. C. Lineberger.

\section{New Directions}

\subsection{Reactions of ions with organic radicals}

Thousands of ion reactions have been studied with stable neutrals and with some atomic radicals in the gas phase. ${ }^{40)}$ In contrast, reactions of ions with polyatomic organic radicals have rarely been studied because of the inherent experimental difficulties. The fundamental chemistry of ion-polyatomic radical reactions, where the charge and radical sites are split between the collision partners, is thus largely unexplored. Ion-organic radical reactions are expected to be important in several environments, including in combustion processes where both positive and negative ions have been identified ${ }^{133)}$ at densities of $10^{8}-10^{10} \mathrm{~cm}^{-3}$. In the interstellar medium more than a hundred organic species have been detected, among them about one-third are radicals or carbenes and several are ions. ${ }^{134)}$ Under very low temperature conditions in space, barrierless ion-radical association and reactions may play a significant role for the formation and evolution of those interstellar species.

Investigations of ion-organic radical reactions are challenging primarily because of the difficulty in generating the radicals cleanly and in high densities. This contrasts with generation of atomic radicals, which can be achieved relatively easily by dissociation of their precursors in a microwave discharge or over a heated metal surface. These atomic sources have been combined with the SIFT ${ }^{135), 136)}$ and Fourier transform ion cyclotron resonance (FT-ICR) ${ }^{137)}$ instruments to study reactions of $\mathrm{H}, \mathrm{N}$, and $\mathrm{O}$ atoms.

For polyatomic radicals, chemical ionization mass spectrometry (CIMS) has begun to use some ion-radical reactions to probe the atmospheric chemistry of alkyl peroxyl $(\mathrm{ROO} \bullet)^{138)-144)}$ and peroxyacetyl $\left(\mathrm{CH}_{3} \mathrm{C}(\mathrm{O}) \mathrm{OO} \bullet\right)$ radicals. ${ }^{145)}$ For example, when $\mathrm{ROO}$ - radicals are undergoing neutral radical chemistry with NO, their concentration changes are monitored by using chemical ionization with $\mathrm{O}_{2}{ }^{+}, \mathrm{O}_{2}{ }^{-}$, or $\mathrm{H}_{3} \mathrm{O}^{+}\left(\mathrm{H}_{2} \mathrm{O}\right)_{n}$. The alkyl peroxyl radicals are prepared by addition of $\mathrm{O}_{2}$ to the corresponding alkyl radicals, which have been generated from suitable precursor molecules by pyrolysis, low-power radio-frequency discharge, or atom reactions. ${ }^{138)-144)}$

Besides those CIMS applications, a versatile method for polyatomic radical generation is expected to open a fertile research field of ion-radical chemistry. We have employed a new approach that combines a supersonic pyrolysis nozzle ${ }^{146)}$ with the SIFT instrument. ${ }^{147), 148)}$ Thermal decomposition of a variety of organic compounds in a pulsed heated nozzle (300-1800 K) produces clean and intense beams of organic radicals or diradicals. ${ }^{149)}$ The high temperature and short residence time in the nozzle $(\approx 30 \mu$ s) ensures clean pyrolysis of the precursor molecules while retaining high conversion efficiencies (60-90\%). The produced radicals are pulsed $(\approx 1.3 \mathrm{~ms}$ in duration, $20-40 \mathrm{~Hz})$ into the high-pressure SIFT flow tube, where both the ions and radicals are quickly thermally equilibrated before the reaction initiates $(\sim 300 \mathrm{~K})$. The ions and radicals are then contained for a moderately long reaction time of about $10 \mathrm{~ms}$. While the ion-radical reaction products can easily be detected and quantified, depletion of the reactant ion is too small to be measured directly under the experimental conditions. However, a simple treatment of pulsed kinetics allows evaluation of the reaction rate constants based on the product yields. ${ }^{147), 148)}$

A simple radical allyl $\left(\mathrm{C}_{3} \mathrm{H}_{5}\right)$ and a diradical orthobenzyne $\left(o-\mathrm{C}_{6} \mathrm{H}_{4}\right)$ have been generated by pyrolysis of allyl iodide $\left(\mathrm{C}_{3} \mathrm{H}_{5} \mathrm{I}+\Delta \rightarrow \mathrm{C}_{3} \mathrm{H}_{5}+\mathrm{I}\right)$ and benzoyl chloride $\left(\mathrm{C}_{6} \mathrm{H}_{5} \mathrm{COCl}+\Delta \rightarrow o-\mathrm{C}_{6} \mathrm{H}_{4}+\mathrm{CO}+\mathrm{Cl}\right)$, respectively, where $\Delta$ represents heat. The strained triple bond in $o^{-}$ benzyne carries a triplet diradical character, which renders the bond highly reactive. These radicals encounter hydronium $\left(\mathrm{H}_{3} \mathrm{O}^{+}\right)$or hydroxide ions $\left(\mathrm{HO}^{-}\right)$in the flow tube. ${ }^{147), 148)}$ The allyl radical and $o$-benzyne react with $\mathrm{H}_{3} \mathrm{O}^{+}$via exothermic proton transfer (Eqs. (26) and (28)) at nearly $100 \%$ efficiencies relative to the ion-radical collision rates. No charged products were observed from allyl $+\mathrm{HO}^{-}$within detection limits (Eq. (27)). The ion-radical encounter might be essentially nonreactive; the heat of deprotonation for the allyl radical is unknown. Alternatively, this finding could result from associative/reactive detachment pathways 
(e.g., $\quad \mathrm{CH}_{2}=\mathrm{CHCH}_{2} \bullet+\mathrm{HO}^{-} \rightarrow \mathrm{CH}_{2}=\mathrm{CHCH}_{2} \mathrm{OH}+\mathrm{e}$ ), which are highly exothermic. The reaction is then analogous to associative detachment of anions with atomic radicals, which is commonly very rapid. 40), 136), 150)

$$
\begin{aligned}
& \mathrm{CH}_{2}=\mathrm{CH}-\dot{\mathrm{C}} \mathrm{H}_{2}+\mathrm{H}_{3} \mathrm{O}^{+} \rightarrow \mathrm{C}_{3} \mathrm{H}_{6}{ }^{+}+\mathrm{H}_{2} \mathrm{O} \\
& \mathrm{CH}_{2}=\mathrm{CH}-\dot{\mathrm{C}} \mathrm{H}_{2}+\mathrm{HO}^{-} \rightarrow \text { no product ions } \\
& +\mathrm{H}_{3} \mathrm{O}^{+} \rightarrow \mathrm{C}_{6} \mathrm{H}_{5}{ }^{+}+\mathrm{H}_{2} \mathrm{O}
\end{aligned}
$$

The reaction of $o$-benzyne with $\mathrm{HO}^{-}$shows an intriguing result. Although abstraction of the most acidic $\alpha$ proton is exothermic by more than $10 \mathrm{kcal} \mathrm{mol}^{-1}$, the observed product $\mathrm{C}_{6} \mathrm{H}_{3}{ }^{-}$(Eq. (29)) accounts for only about $15 \%$ of the collision. This is an unexpected result because exothermic proton abstraction is usually a barrierless process (Fig. 3a) that takes place at every collision. The observation may be rationalized by competing associative detachment; the $\mathrm{HO}^{-}$ion could add to the triple bond to form $o$-hydroxy phenyl anion $\left(o-\mathrm{C}_{6} \mathrm{H}_{4} \mathrm{OH}^{-}\right)$, which could subsequently lose the electron because of the large reaction exothermicity far exceeding the binding energy. ${ }^{147), 148)}$ Thus, this is a novel fundamental reaction channel for $\mathrm{HO}^{-}$ions that is facilitated by the high energy content in $o$-benzyne. Future experiments include trapping of detached electrons with electron scavengers such as $\mathrm{SF}_{6}$. Other radicals methyl and phenyl as well as diradical cyclopropenylidene $\left(c-\mathrm{C}_{3} \mathrm{H}_{2}\right)$ are among the reactants that can be generated and studied using the SIFTsupersonic pyrolysis technique.

\subsection{Chemical ionization mass spectrometry}

Compared to other ionization methods such as electron impact or hollow cathode discharge, chemical ionization is a soft ionization that bestows charge to neutral molecules with minimal fragmentation. ${ }^{151)}$ Chemical ionization mass spectrometry, which conveniently uses proton transfer/abstraction reactions of $\mathrm{H}_{3} \mathrm{O}^{+}$or $\mathrm{HO}^{-}$reagent ions in flow-drift tube reactors, ${ }^{152), 153)}$ has become increasingly popular for highly sensitive, online detection of a variety of volatile organic compounds (VOCs). We utilized $\mathrm{H}_{3} \mathrm{O}^{+}$proton transfer reactions to measure aldehydes from human cancer cells ${ }^{154)-156)}$ and VOCs from fermenting bacteria. ${ }^{157)} \mathrm{We}$ also demonstrated that negative ion chemistry with $\mathrm{HO}^{-}$is an alternative tool to monitor plant emission of VOCs $^{158)-160)}$ and to detect small, volatile nitroalkane explosives. ${ }^{107)}$ These analytical applications and bio/ medico-chemical implications, as well as ion-molecule chemistry of interstellar relevance, will be discussed elsewhere. ${ }^{161)}$

A new reaction scheme of CIMS interest is given here. While reactions with $\mathrm{H}_{3} \mathrm{O}^{+}$and $\mathrm{HO}^{-}$are facile and versatile in converting a majority of organic species to respective $[\mathrm{M}+\mathrm{H}]^{+}$and $[\mathrm{M}-\mathrm{H}]^{-}$product ions, identification of isobaric products is usually a difficult task. CID and H/D exchange reactions may be used to differentiate some product isomers, for example, deprotonated acetone versus propanal. ${ }^{157)}$ Another approach

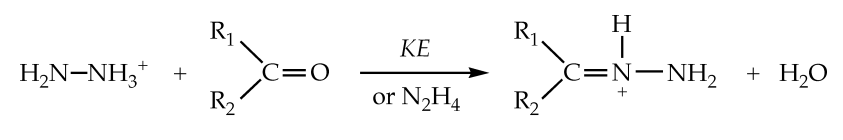

19

Scheme 11. Gas phase reaction of hydrazinium ion with carbonyl to form protonated hydrazone.

to structure identification is to devise a CI scheme with chemical specificity. In analogy to acid-catalyzed, solution phase chemistry of hydrazine and its derivatives, we have shown that reactions of protonated hydrazine $\left(\mathrm{N}_{2} \mathrm{H}_{5}{ }^{+}\right)$can be used to specifically detect carbonyl compounds in the gas phase. ${ }^{162), 163)} \mathrm{N}_{2} \mathrm{H}_{5}{ }^{+}$reacts with a carbonyl compound and forms a characteristic product, protonated hydrazone (Scheme 11). This reaction, while exothermic, is slow and observed only when excess neutral $\mathrm{N}_{2} \mathrm{H}_{4}$ molecules are present. ${ }^{163)}$ Further, formation of hydrazone is dramatically enhanced at high ion kinetic energies in the drift field. ${ }^{162)}$ Additional experiments using deuterated $\mathrm{N}_{2} \mathrm{D}_{4}$ and density functional calculations ${ }^{163)}$ have identified the origin of the slowness of the reaction: Transition states for the bimolecular reaction (Scheme 11) present kinetic and energetic barriers that can be overcome by translational and/or chemical activation. The energies for the strained transition states are likely to be lowered in the presence of a second $\mathrm{N}_{2} \mathrm{H}_{4}$ molecule within the intermediate complex. ${ }^{163)}$ The fundamental studies have provided an important guideline for this CIMS scheme to be widely used for future applications.

\section{Conclusions}

Various aspects of fundamental gas phase ion chemistry have been discussed. The SIFT technique is a versatile tool with which to study the kinetics and mechanisms of ion reactions in a very clean, truly thermal environment. This feature has been fully utilized, for example, to reveal nonstatistical behaviors in $\mathrm{S}_{\mathrm{N}} 2$ kinetics and to prove otherwise controversial $\mathrm{E}_{\mathrm{CO}} 2$ reactions of biological interest, as well as address classical questions in organic chemistry and explore new schemes for CIMS applications. By incorporating LIF and triple quadrupole detections and through a powerful coupling with NIPES experiments, the SIFT technique has continued to provide detailed information on ion structure, energetics, and dynamics. These results have stimulated theoretical interest as well. We have shown that a hyperthermal radical source can be used with the SIFT instrument for studies of ion-polyatomic radical reactions. ESI sources are also expected to be useful for loading ions of nonvolatile species in the flow tube. These technical developments will further extend the research horizon with the SIFT technique.

\section{Acknowledgments}

The author acknowledges Prof. Stephen R. Leone for introduction to the field of gas-phase ion chemical dynamics as studied by SIFT-LIF, and Profs. Venonica M. Bierbaum and Charles H. DePuy during the subsequent thermochemical and physical organic studies using more classical SIFT designs. The author thanks Profs. W. Carl Lineberger, G. Barney Ellison, Stephen J. Blank- 
sby, Weston Thatcher Borden, and Dr. Carleton J. Howard, among others, for the work in this article. Invitation and encouragement from Prof. Kenzo Hiraoka to write this article is gratefully appreciated. The work was supported by the National Science Foundation (CHE-0349937).

\section{References}

1) V. M. Bierbaum, "Flow Tubes," in Encyclopedia of Mass Spectrometry, ed. by M. L. Gross and R. Caprioli, Elsevier, Amsterdam (2003), Vol. 1, Theory and Ion Chemistry, p. 98.

2) D. Smith and N. G. Adams, Int. J. Mass Spectrom. Ion Phys., 21, 349 (1976).

3) D. Smith and N. G. Adams, "Recent Advances in Flow Tubes: Measurement of Ion-Molecule Rate Coefficients and Product Distribution," in Gas Phase Ion Chemistry, ed. by M. T. Bowers, Academic Press, New York (1979), Vol. 1, p. 1.

4) D. Smith and N. G. Adams, Adv. Atom. Mol. Phys., 24, 1 (1988).

5) S. T. Graul and R. R. Squires, Mass Spectrom. Rev., 7, 263 (1988).

6) S. Kato, M. J. Frost, V. M. Bierbaum, and S. R. Leone, Rev. Sci. Instrum., 64, 2808 (1993).

7) P. J. Marinelli, J. A. Paulino, L. S. Sunderlin, P. G. Wenthold, J. C. Poutsma, and R. R. Squires, Int. J. Mass Spectrom. Ion Processes, 130, 89 (1994).

8) J. M. Van Doren, S. E. Barlow, C. H. DePuy, and V. M. Bierbaum, Int. J. Mass Spectrom. Ion Processes, 81, 85 (1987).

9) H. S. Lee and V. M. Bierbaum, J. Chem. Phys., 101, 9513 (1994).

10) S. Kato, S. R. Leone, V. M. Bierbaum, and L. H. Haber, AIAA 2004-1015, 1 (2004).

11) S. Kato, "Product State Distributions/Ion Spectroscopy," in Encyclopedia of Mass Spectrometry, ed. by M. L. Gross and R. Caprioli, Elsevier, Amsterdam (2003), Vol. 1, Theory and Ion Chemistry, p. 276.

12) E. E. Ferguson, J. Phys. Chem., 90, 731 (1986).

13) K. Kuratani and S. Tsuchiya, "Shock Waves in Chemistry and Physics," Shokabo, Tokyo (1968).

14) J. T. Yardley, "Introduction to Molecular Energy Transfer," Academic Press, New York (1980).

15) S. R. Leone, J. Phys. Chem. Ref. Data, 11, 953 (1982).

16) S. Kato, V. M. Bierbaum, and S. R. Leone, Int. J. Mass Spectrom. Ion Processes, 149/150, 469 (1995).

17) S. Kato, J. A. de Gouw, C. D. Lin, V. M. Bierbaum, and S. R. Leone, J. Chem. Phys., 105, 5455 (1996).

18) S. Kato, M. J. Frost, V. M. Bierbaum, and S. R. Leone, Can. J. Chem., 72, 625 (1994).

19) M. J. Frost, S. Kato, V. M. Bierbaum, and S. R. Leone, J. Chem. Phys., 98, 5993 (1993).

20) M. J. Frost, S. Kato, V. M. Bierbaum, and S. R. Leone, J. Chem. Phys., 100, 6359 (1994).

21) S. Kato, V. M. Bierbaum, and S. R. Leone, J. Phys. Chem. A, 102, 6659 (1998).

22) M. J. Frost, S. Kato, V. M. Bierbaum, and S. R. Leone, Chem. Phys., 231, 145 (1998).

23) J. A. de Gouw, L. N. Ding, M. J. Frost, S. Kato, V. M. Bierbaum, and S. R. Leone, Chem. Phys. Lett., 240, 362 (1995).

24) E. A. Gislason and E. E. Ferguson, J. Chem. Phys., 87, 6474 (1987).

25) E. E. Ferguson, R. Richter, and W. Lindinger, J. Chem. Phys., 89, 1445 (1988).

26) A. Tachibana, K. Nakamura, T. Yano, Y. Sugiyama, and
S. Tanimura, J. Phys. Chem. A, 103, 5749 (1999).

27) K. Hiraoka and G. Nakajima, J. Chem. Phys., 88, 7709 (1988).

28) K. Norwood, G. Luo, and C. Y. Ng, J. Chem. Phys., 91, 849 (1989).

29) K. Sohlberg, Chem. Phys., 246, 307 (1999).

30) T. Wyttenbach and M. T. Bowers, J. Phys. Chem., 97, 9573 (1993).

31) H. Villinger, P. Lukac, F. Howorka, E. Alge, H. Ramler, and W. Lindinger, Czech. J. Phys. B, 31, 832 (1981).

32) T. Kato, K. Tanaka, and I. Koyano, J. Chem. Phys., 77, 834 (1982).

33) S. Kato, J. A. de Gouw, C. D. Lin, V. M. Bierbaum, and S. R. Leone, Chem. Phys. Lett., 256, 305 (1996).

34) M. Lipeles, J. Chem. Phys., 51, 1252 (1969).

35) K. Hasegawa, K. Doi, K. Nakamura, and A. Tachibana, Mol. Phys., 101, 295 (2003).

36) W. J. Knott, D. Proch, and K. L. Kompa, J. Chem. Phys., 110, 9426 (1999).

37) I. Koyano, K. Tanaka, T. Kato, and S. Suzuki, Faraday Discuss. Chem. Soc., 84, 265 (1987).

38) J. K. Laerdahl and E. Uggerud, Int. J. Mass Spectrom., 214, 277 (2002).

39) S. E. Barlow, J. M. Van Doren, and V. M. Bierbaum, J. Am. Chem. Soc., 110, 7240 (1988).

40) Y. Ikezoe, S. Matsuoka, M. Takebe, and A. Viggiano, "Gas Phase Ion-Molecule Reaction Rate Constants Through 1986," Maruzen, Tokyo (1987).

41) F. E. Wilkinson, M. Peschke, J. E. Szulejko, and T. B. McMahon, Int. J. Mass Spectrom. Ion Processes, 175, 225 (1998).

42) C. Li, P. Ross, J. E. Szulejko, and T. B. McMahon, J. Am. Chem. Soc., 118, 9360 (1996).

43) W. N. Olmstead and J. I. Brauman, J. Am. Chem. Soc., 99, 4219 (1977).

44) M. N. Glukhovtsev, A. Pross, H. B. Schlegel, R. D. Bach, and L. Radom, J. Am. Chem. Soc., 118, 11258 (1996).

45) V. F. DeTuri, P. A. Hintz, and K. M. Ervin, J. Phys. Chem., 101, 5969 (1997)

46) G. Li and W. L. Hase, J. Am. Chem. Soc., 121, 7124 (1999).

47) M. N. Glukhovtsev, A. Pross, and L. Radom, J. Am. Chem. Soc., 117, 2024 (1995).

48) L. Sun, W. L. Hase, and K. Song, J. Am. Chem. Soc., 123, 5753 (2001).

49) L. Sun, K. Song, and W. L. Hase, Science, 296, 875 (2002).

50) K. M. Ervin, Int. J. Mass Spectrom., 185/186/187, 343 (1999).

51) M. L. Chabinyc, S. L. Craig, C. K. Regan, and J. I. Brauman, Science, 279, 1882 (1998).

52) P. J. Robinson and K. A. Holbrook, "Unimolecular Reactions," Wiley, New York (1972).

53) T. Baer and W. L. Hase, "Unimolecular Reaction Dynamics," Oxford University Press, New York (1996).

54) A. A. Viggiano and R. A. Morris, J. Phys. Chem., 100, 19227 (1996).

55) D. S. Tonner and T. B. McMahon, J. Am. Chem. Soc., 122, 8783 (2000).

56) S. T. Graul and M. T. Bowers, J. Am. Chem. Soc., 116, 3875 (1994).

57) H. Wang, G. H. Peslherbe, and W. L. Hase, J. Am. Chem. Soc., 116, 9644 (1994).

58) R. Wester, A. E. Bragg, A. V. Davis, and D. M. Neumark, J. Chem. Phys., 119, 10032 (2003).

59) "Isotope Effects in Gas-Phase Chemistry," ACS Symposium Series, Vol. 502, ed. by J. A. Kaye, American Chemical Society, Washington, DC (1992).

60) R. A. Poirier, Y. Wang, and K. C. Westaway, J. Am. Chem. 
Soc., 116, 2526 (1994).

61) S. Kato, G. E. Davico, H. S. Lee, C. H. DePuy, and V. M. Bierbaum, Int. J. Mass Spectrom., 210/211, 223 (2001).

62) L. A. Angel and K. M. Ervin, J. Phys. Chem. A, 105, 4042 (2001).

63) H. Tachikawa and M. Igarashi, Chem. Phys. Lett., 303, 81 (1999).

64) W.-P. Hu and D. G. Truhlar, J. Am. Chem. Soc., 117, 10726 (1995).

65) H. Wang and W. L. Hase, J. Am. Chem. Soc., 117, 9347 (1995).

66) S. S. Glad and F. Jensen, J. Am. Chem. Soc., 119, 227 (1997).

67) G. E. Davico and V. M. Bierbaum, J. Am. Chem. Soc., 122, 1740 (2000).

68) A. A. Viggiano, S. T. Arnold, and R. A. Morris, Int. Rev. Phys. Chem., 17, 147 (1998).

69) K. Takashima and J. M. Riveros, Mass Spectrom. Rev., 17, 409 (1998).

70) R. A. J. O'Hair, G. E. Davico, J. Hacaloglu, T. T. Dang, C. H. DePuy, and V. M. Bierbaum, J. Am. Chem. Soc., 116, 3609 (1994).

71) W.-P. Hu and D. G. Truhlar, J. Am. Chem. Soc., 116, 7797 (1994).

72) H. Tachikawa, J. Phys. Chem. A, 104, 497 (2000).

73) H. Tachikawa, J. Phys. Chem. A, 105, 1260 (2001).

74) S. Kato, J. Hacaloglu, G. E. Davico, C. H. DePuy, and V. M. Bierbaum, J. Phys. Chem. A, 108, 9887 (2004).

75) K. Hiraoka and P. Kebarle, J. Am. Chem. Soc., 98, 6119 (1976).

76) H. S. Lee, V. M. Bierbaum, and C. H. DePuy, Int. J. Mass Spectrom. Ion Processes, 167/168, 587 (1997).

77) J. W. d. M. Carneiro, P. v. R. Schleyer, M. Saunders, R. Remington, H. F. Shaefer, III, A. Rauk, and T. S. Sorensen, J. Am. Chem. Soc., 116, 3483 (1994).

78) C. H. DePuy and S. Kato, "H/D Exchange Reactions/ Organic Ion Chemistry," in Encyclopedia of Mass Spectrometry, ed. by M. L. Gross and R. Caprioli, Elsevier, Amsterdam (2003), Vol. 1, Theory and Ion Chemistry, p. 670.

79) S. Kato, C. H. DePuy, S. Gronert, and V. M. Bierbaum, J. Am. Soc. Mass Spectrom., 10, 840 (1999).

80) S. Kato, T. T. Dang, S. E. Barlow, C. H. DePuy, and V. M. Bierbaum, Int. J. Mass Spectrom., 195/196, 625 (2000).

81) A. T. Pudzianowski, J. Chem. Phys., 102, 8029 (1995).

82) M. Masamura and S. Ikuta, J. Comput. Chem., 20, 1138 (1999).

83) D. A. Weil and D. A. Dixon, J. Am. Chem. Soc., 107, 6859 (1985).

84) E. Erdik and M. Ay, Chem. Rev., 89, 1947 (1989).

85) R. Ulbrich, M. Famulok, F. Bosold, and G. Boche, Tetrahedron Lett., 31, 1689 (1990).

86) J. S. Helmick, K. A. Martin, J. L. Heinrich, and M. Novak, J. Am. Chem. Soc., 113, 3459 (1991).

87) P. Beak and J. Li, J. Am. Chem. Soc., 113, 2796 (1991).

88) M. Bühl and H. F. Schaefer III, J. Am. Chem. Soc., 115, 9143 (1993)

89) M. Bühl and H. F. Schaefer III, J. Am. Chem. Soc., 115, 364 (1993).

90) R. Gareyev, S. Kato, and V. M. Bierbaum, J. Am. Soc. Mass Spectrom., 12, 139 (2001).

91) M. N. Glukhovtsev, A. Pross, and L. Radom, J. Am. Chem. Soc., 117, 9012 (1995).

92) C. H. DePuy, S. Gronert, A. Mullin, and V. M. Bierbaum, J. Am. Chem. Soc., 112, 8650 (1990).

93) S. Gronert, C. H. DePuy, and V. M. Bierbaum, J. Am. Chem. Soc., 113, 4009 (1991).
94) S. Gronert, Acc. Chem. Res., 36, 848 (2003).

95) S. J. Blanksby, G. B. Ellison, V. M. Bierbaum, and S. Kato, J. Am. Chem. Soc., 124, 3196 (2002).

96) N. Kornblum and H. E. DeLaMare, J. Am. Chem. Soc., 73, 880 (1951).

97) R. Hofmann, H. Hübner, G. Just, L. Krätzsch, A. K. Litkowez, W. Pritzkow, W. Rolle, and M. Wahren, J. Prakt. Chem., 37, 102 (1968).

98) L. J. Marnett, Carcinogenesis, 21, 361 (2000).

99) S. H. Lee, T. Oe, and I. A. Blair, Science, 292, 2083 (2001).

100) S. J. Blanksby, S. Kato, V. M. Bierbaum, and G. B. Ellison, Aust. J. Chem., 56, 459 (2003).

101) D. K. MacMillan and R. C. Murphy, J. Am. Soc. Mass Spectrom., 6, 1190 (1995).

102) S. J. Blanksby, T. M. Ramond, G. E. Davico, M. R. Nimlos, S. Kato, V. M. Bierbaum, W. C. Lineberger, G. B. Ellison, and M. Okumura, J. Am. Chem. Soc., 123, 9585 (2001).

103) T. M. Ramond, S. J. Blanksby, S. Kato, V. M. Bierbaum, G. E. Davico, R. L. Schwartz, W. C. Lineberger, and G. B. Ellison, J. Phys. Chem. A, 106, 9641 (2002).

104) J. Berkowitz, G. B. Ellison, and D. Gutman, J. Phys. Chem., 98, 2744 (1994).

105) M. W. Chase, Jr., "NIST-JANAF Thermochemical Tables," American Chemical Society, Washington, DC (1998).

106) E. P. Clifford, P. G. Wenthold, W. C. Lineberger, G. A. Petersson, K. M. Broadus, S. R. Kass, S. Kato, C. H. DePuy, V. M. Bierbaum, and G. B. Ellison, J. Phys. Chem., 102, 7100 (1998).

107) S. Kato, K. E. Carrigan, C. H. DePuy, and V. M. Bierbaum, Eur. J. Mass Spectrom., 10, 225 (2004).

108) A. J. Gianola, T. Ichino, R. L. Hoenigman, S. Kato, V. M. Bierbaum, and W. C. Lineberger, J. Phys. Chem. A, 108, 10326 (2004).

109) R. Engelke, W. L. Earl, and C. M. Rohlfing, Int. J. Chem. Kinet., 18, 1205 (1986).

110) Y. A. Gruzdkov and Y. M. Gupta, J. Phys. Chem. A, 102, 2322 (1998).

111) E. Woods III, Y. Dessiaterik, R. E. Miller, and T. Baer, J. Phys. Chem. A, 105, 8273 (2001).

112) J. E. Bartmess, J. A. Scott, and R. T. McIver Jr., J. Am. Chem. Soc., 101, 6046 (1979).

113) R. J. Bartlett, Chemistry \& Industry, 21 February, 140 (2000).

114) M. T. Nguyen and T.-K. Ha, Chem. Phys. Lett., 335, 311 (2001).

115) V. Benin, P. Kaszynski, and J. G. Radziszewski, J. Org. Chem., 67, 1354 (2002).

116) A. Vij, J. G. Pavlovich, W. W. Wilson, V. Vij, and K. O. Christe, Angew. Chem. Int. Ed., 41, 3051 (2002).

117) H. Östmark, S. Wallin, T. Brinck, P. Carlqvist, R. Claridge, E. Hedlund, and L. Yudina, Chem. Phys. Lett., 379, 539 (2003).

118) R. N. Butler, J. C. Stephens, and L. A. Burke, Chem. Commun., 1016 (2003).

119) G. I. Fray and R. G. Saxton, "The Chemistry of Cyclooctatetraene and Its Derivatives," Cambridge University Press, New York (1978).

120) L. A. Paquette, Acc. Chem. Res., 26, 57 (1993).

121) P. G. Wenthold, D. A. Hrovat, W. T. Borden, and W. C. Lineberger, Science, 272, 1456 (1996).

122) S. Kato, H. S. Lee, R. Gareyev, P. G. Wenthold, W. C. Lineberger, C. H. DePuy, and V. M. Bierbaum, J. Am. Chem. Soc., 119, 7863 (1997).

123) J. W. Denault, G. Chen, and R. G. Cooks, J. Am. Soc. Mass Spectrom., 9, 1141 (1998).

124) T. M. Miller, A. A. Viggiano, and A. E. S. Miller, J. Phys. 
Chem. A, 106, 10200 (2002).

125) L.-M. Frutos, O. Castaño, and M. Merchán, J. Phys. Chem. A, 107, 5472 (2003).

126) S. Kato, R. Gareyev, C. H. DePuy, and V. M. Bierbaum, J. Am. Chem. Soc., 120, 5033 (1998).

127) T. Bally, S. Chai, M. Neuenschwander, and Z. Zhu, J. Am. Chem. Soc., 119, 1869 (1997).

128) M. Tsuji, personal communication.

129) J. Lee and J. J. Grabowski, Chem. Rev., 92, 1611 (1992).

130) M. Born, S. Ingemann, and N. M. M. Nibbering, Mass Spectrom. Rev., 16, 181 (1997).

131) Y. Guo and J. J. Grabowski, Int. J. Mass Spectrom. Ion Processes, 117, 299 (1992).

132) R. L. Hoenigman, S. Kato, W. T. Borden, and V. M. Bierbaum, Int. J. Mass Spectrom., 241, 149 (2005).

133) A. N. Hayhurst and D. B. Kittelson, Combust. Flame, 31, 37 (1978).

134) M. C. McCarthy and P. Thaddeus, Chem. Soc. Rev., 30, 177 (2001).

135) V. Le Page, H. S. Lee, V. M. Bierbaum, and T. P. Snow, NASA Conference Publication, 3343, 125 (1996).

136) C. Barckholtz, T. P. Snow, and V. M. Bierbaum, Astrophys. J., 547, L171 (2001).

137) P. A. Demirev, Rapid Commun. Mass Spectrom., 14, 777 (2000).

138) P. W. Villalta, L. G. Huey, and C. J. Howard, J. Phys. Chem., 99, 12829 (1995).

139) P. W. Villalta and C. J. Howard, J. Phys. Chem., 100, 13624 (1996).

140) J. Eberhard and C. J. Howard, J. Phys. Chem. A, 101, 3360 (1997).

141) K. W. Scholtens, B. M. Messer, C. D. Cappa, and M. J. Elrod, J. Phys. Chem. A, 103, 4378 (1999).

142) D. L. Ranschaert, N. J. Schneider, and M. J. Elrod, J. Phys. Chem. A, 104, 5758 (2000).

143) J. M. Chow, A. M. Miller, and M. J. Elrod, J. Phys. Chem. A, 107, 3040 (2003).

144) D. Hanson, J. Orlando, B. Noziere, and E. Kosciuch, Int. J. Mass Spectrom., 239, 147 (2004)

145) J. Eberhard, P. W. Villalta, and C. J. Howard, J. Phys. Chem., 100, 993 (1996).

146) J. A. Blush, H. Clauberg, D. W. Kohn, D. W. Minsek, X.
Zhang, and P. Chen, Acc. Chem. Res., 25, 385 (1992).

147) X. Zhang, V. M. Bierbaum, G. B. Ellison, and S. Kato, J. Chem. Phys., 120, 3531 (2004).

148) X. Zhang, S. Kato, V. M. Bierbaum, M. R. Nimlos, and G. B. Ellison, J. Phys. Chem. A., 108, 9733 (2004).

149) X. Zhang, A. V. Friderichsen, S. Nandi, G. B. Ellison, D. E. David, J. T. McKinnon, T. G. Lindeman, D. C. Dayton, and M. R. Nimlos, Rev. Sci. Instrum., 74, 3077 (2003).

150) F. C. Fehsenfeld, "Associative Detachment," in Interactions between Ions and Molecules, ed. by P. Ausloos, Plenum, New York (1975), p. 387.

151) A. G. Harrison, "Chemical Ionization Mass Spectrometry," CRC Press, Boca Raton (1992).

152) D. Smith and P. Spanel, Int. Rev. Phys. Chem., 15, 231 (1996).

153) W. Lindinger, A. Hansel, and A. Jordan, Int. J. Mass Spectrom. Ion Processes, 173, 191 (1998).

154) S. Kato, P. J. Burke, D. J. Fenick, D. J. Taatjes, V. M. Bierbaum, and T. H. Koch, Chem. Res. Toxicol., 13, 509 (2000).

155) S. Kato, P. J. Burke, T. H. Koch, and V. M. Bierbaum, Anal. Chem., 73, 2992 (2001).

156) S. Kato, G. C. Post, V. M. Bierbaum, and T. H. Koch, Anal. Biochem., 305, 251 (2002).

157) T. G. Custer, W. P. Wagner, S. Kato, V. M. Bierbaum, and R. Fall, Biotechnol. Progress, 19, 1355 (2003).

158) T. G. Custer, S. Kato, R. Fall, and V. M. Bierbaum, Geophys. Res. Lett., 27, 3849 (2000).

159) R. Fall, T. G. Custer, S. Kato, and V. M. Bierbaum, Atmospheric Environment, 35, 1713 (2001).

160) T. G. Custer, S. Kato, R. Fall, and V. M. Bierbaum, Int. J. Mass Spectrom., 223/224, 427 (2003).

161) S. Kato and V. M. Bierbaum (in preparation).

162) G. C. Morrison and C. J. Howard, Int. J. Mass Spectrom., 210/211, 503 (2001)

163) T. G. Custer, S. Kato, V. M. Bierbaum, C. J. Howard, and G. C. Morrison, J. Am. Chem. Soc., 126, 2744 (2004).

Keywords: Gas phase ion chemistry, Selected ion flow tube, Vibrationally state-selected chemistry, Organic reaction mechanism, Thermochemistry 